\title{
A Three-step Methodology for Dimensional Tolerance Synthesis of Parallel Manipulators
}

\author{
Alexandre Goldsztejn ${ }^{\mathrm{a}, *}$, Stéphane Caro $^{\mathrm{a}}$, Gilles Chabert ${ }^{\mathrm{b}}$ \\ ${ }^{a}$ IRCCyN, CNRS, Nantes, France \\ ${ }^{b}$ LINA, Ecole des Mines de Nantes, Nantes, France
}

\begin{abstract}
Computing the maximal pose error given an upper bound on model parameters uncertainties, called perturbations in this paper, is challenging for parallel robots, mainly because the direct kinematic problem has several solutions, which become unstable in the vicinity of parallel singularities. In this paper, a local uniqueness hypothesis that allows safely computing pose error upper bounds using nonlinear optimization is proposed. This hypothesis, together with a corresponding maximal allowed perturbation domain and a certified crude pose error upper bound valid over the complete workspace, will be proved numerically using a parametric version of Kantorovich theorem and certified nonlinear global optimization. Then, approximate linearizations are used in order to determine approximated tolerances reaching a prescribed maximal pose error over a given workspace. Those tolerances are finally verified using optimal pose error upper bounds, which are computed using global optimization techniques. Two illustrative examples are studied in order to highlight the contributions of the paper.
\end{abstract}

Keywords: Certified tolerance synthesis, parallel manipulators, parametric Kantorovich theorem

\section{Introduction}

For two decades, parallel manipulators have attracted the attention of more and more researchers who consider them as valuable alternative design for robotic mechanisms. Parallel Kinematics Machines (PKM) offer essential advantages over their serial counterparts such as lower moving masses, higher stiffness and payload-to-weight ratio, higher natural frequencies and better dynamic performance.

\footnotetext{
* Corresponding author

Email addresses: Alexandre.Goldsztejn@irccyn.ec-nantes.fr (Alexandre Goldsztejn), Stephane.Caro@irccyn.ec-nantes.fr (Stéphane Caro), Gilles.Chabert@mines-nantes.fr (Gilles Chabert)
}

Preprint submitted to Elsevier

March 17, 2016 
However, PKM are not necessarily more accurate than their serial counterparts. Indeed, even if the dimensional variations can be compensated with PKM, they can also be amplified contrary to with their serial counterparts. Wang et al. 1] studied the effect of manufacturing tolerances on the accuracy of a Stewart platform. Kim et al. 2] used a forward error bound analysis to find the error bound of the end-effector of a Stewart platform when the error bounds of the joints are given, and an inverse error bound analysis to determine those of the joints for the given error bound of the end-effector. Kim and Tsai [3] studied the effect of misalignment of linear actuators of a three Degree-of-Freedom (DOF) translational parallel manipulator on the motion of its moving platform. Han et al. 4] used a kinematic sensitivity analysis method to explain the gross motions of a 3-UPU parallel mechanism, and showed that it is highly sensitive to certain minute clearances. Fan et al. [5] analyzed the sensitivity of the 3-PRS parallel kinematic spindle platform of a serial-parallel machine tool. Verner et al. 6] presented a new method for optimal calibration of PKM based on the exploitation of the least error sensitive regions in their workspace and geometric parameters space. As a matter of fact, they used a Monte Carlo simulation to determine and map the sensitivities to geometric parameters. Moreover, Caro et al. [7] developed a tolerance synthesis method for mechanisms based on a robust design approach. Ryu et al. derived a volumetric error model and a total error transformation matrix from a differential inverse kinematic equation, which includes all kinematic error sources [8. Liu et al. reported an approach of geometric error modeling for lower mobility manipulators by explicitly separating the compensatable and uncompensatable error sources affecting the pose accuracy [9. Briot and Bonev proposed a simple method based on a detailed error analysis of 3-DOF planar parallel robots that brings valuable understanding of the problem of error amplification [10. Rolland used algebraic tools in order to compute an upper bound of the moving platform pose error for a Gough-Stewart platform while considering geometric and numerical errors [11]. Patel and Ehmann analyzed the volumetric error of a Gough-Stewart platform too [12.

During the early design process of engineering systems, the analysis of the performance sensitivity to uncertainties is an important task. High sensitivity to parameters that are inherently noisy can lead to poor, or unexpected performance. For that reason, it is important to analyze the sensitivity of their performance to variations in their geometric parameters and to determine the optimal dimensional tolerances.

To this end, some indices such as the dexterity and the manipulability have been used to evaluate the sensitivity of robots performance to variations in their actuated joints [13, 14, 15]. However, they are not suitable for the evaluation of this sensitivity to other types of uncertainty such as variations in geometric parameters. Two indices were proposed in [16] to evaluate the sensitivity of the end-effector pose (position + orientation) of the Orthoglide 3-axis, a 3DOF translational PKM, to variations in its design parameters. In the same vein, four 3-RPR planar parallel manipulators (PPMs) were compared in [17] based on the sensitivity of their performance to variations in their geometric 
parameters. In [18, an interval linearization method is used for the sensitivity analysis of some parallel manipulators. However, the foregoing research works do not deal with the tolerance synthesis of parallel manipulators, which is a critical issue.

In the present paper, we overcome two lacks in the literature: First, a fully rigorous methodology is proposed to compute a certified upper bound for the pose error due to bounded uncertainties in the model parameters of a PKM throughout its workspace. Second, a methodology is proposed for the tolerance synthesis of PKM, aiming at synthesizing the largest tolerances while keeping the pose error of the moving-platform below a given limit. The proposed tolerance synthesis method is composed of three steps:

Step 1 A rigorous parametric pose error upper bound $\epsilon(\mathbf{p})$ is computed, which depends on the value of the perturbation $\mathbf{p}$, together with a perturbation domain $\mathcal{P}$ where this upper bound is valid. Both are computed using Kantorovich theorem, where Kantorovich constants are evaluated over the full manipulator workspace using certified nonlinear global optimization.

Step 2 Since the previous upper bound is pessimistic, its usage for tolerance synthesis may lead to some over design (i.e., too small tolerances are designed leading to a better accuracy than the required one). Therefore, a non rigorous linearization of the maximum pose error in the workspace is proposed and used for synthesizing approximate tolerances.

Step 3 A rigorous sharp pose error upper bound is finally computed for the tolerance synthesized at Step 2 using certified nonlinear global optimization. The cruder upper bound computed at Step 1 is necessary to make this problem provably consistent.

Step 2 is actually optional: An accurate linear approximation can be obtained by building a linear model using the sharp error upper bound computed at Step 3 for different tolerances. However, the problem to be solved at Step 3 is more difficult than ones to be solved at Step 2, therefore starting with the linear approximation provided by Step 2 can turn out to be more efficient.

The paper is organized as follows. Step 3 motivates the necessity of Step 1, and is therefore first detailed in Section 3. A uniqueness hypothesis is introduced in order to compute a certified sharp pose error upper bound over a given workspace by solving a nonlinear optimization problem. Step 1 is the central contribution of the paper, and is addressed in Section 4. A parametric version of Kantorovich theorem is proposed, which provides both a maximal perturbation domain for which this uniqueness hypothesis holds, and a crude certified pose error upper bound valid inside this perturbation domain as well as in the manipulator workspace. Step 2 is finally developed in Section 5 An approximate linearization of the maximal pose error of the moving-platform in the workspace is proposed, which allows performing some approximate tolerance synthesis. These approximate tolerances can finally be corrected using the results obtained in Section 3 . Two illustrative examples are given in Section 6 
in order to highlight the potential and limits of the approach. The two examples deal with the tolerance synthesis of a RPRPR parallel manipulator and a 3-R $\underline{P R}$ parallel manipulator with a fixed orientation of this moving-platform, respectively.

\section{Notations}

We use the $\infty$-norm throughout the paper. Let $B(\mathbf{x}, \epsilon)$ be the open ball $\left\{\mathbf{y} \in \mathbb{R}^{k}:\|\mathbf{y}-\mathbf{x}\|<\epsilon\right\}, \bar{B}(\mathbf{x}, \epsilon)$ be the closed ball $\left\{\mathbf{y} \in \mathbb{R}^{k}:\|\mathbf{y}-\mathbf{x}\| \leq \epsilon\right\}$, and for short $\bar{B}_{\epsilon}:=\bar{B}(\mathbf{0}, \epsilon)$.

\section{Definitions related to perturbed kinematic models}

We consider a kinematic model $\mathbf{f}(\mathbf{x}, \mathbf{q}, \mathbf{p})=\mathbf{0}$ of a non-redundant parallel manipulator, where $\mathbf{f}: \mathbb{R}^{n} \times \mathbb{R}^{n^{\prime}} \times \mathbb{R}^{m} \rightarrow \mathbb{R}^{n}$, x being the pose, $\mathbf{q}$ the actuated joint coordinates and $\mathbf{p}$ a perturbation vector associated to some uncertain model parameters, so that the nominal model is $\mathbf{f}(\mathbf{x}, \mathbf{q}, \mathbf{0})=\mathbf{0}$. If $n^{\prime}>n$ then the robot is over-actuated, which can be handled by the method proposed in this paper, although experiments presented in Section 6 are restricted to $n=n^{\prime}$. We also denote this nominal model by $\mathbf{f}(\mathbf{x}, \mathbf{q}):=\mathbf{f}(\mathbf{x}, \mathbf{q}, \mathbf{0})$.

Example 1. The nominal model for a $R \underline{P} R \underline{P} R$ is

$$
\begin{aligned}
& \left(x_{1}+1\right)^{2}+x_{2}^{2}-l_{1}^{2}=0 \\
& \left(x_{1}-1\right)^{2}+x_{2}^{2}-l_{2}^{2}=0 .
\end{aligned}
$$

Considering perturbations $p_{i}, i \in\{1, \ldots, 4\}$, on the position of the fixed revolute joints, and $p_{i}, i \in\{5,6\}$, on the lengths of the actuated prismatic joints gives rise to

$$
\begin{aligned}
& \left(x_{1}+1+p_{1}\right)^{2}+\left(x_{2}+p_{2}\right)^{2}-\left(l_{1}+p_{5}\right)^{2}=0 \\
& \left(x_{1}-1+p_{3}\right)^{2}+\left(x_{2}+p_{4}\right)^{2}-\left(l_{2}+p_{6}\right)^{2}=0
\end{aligned}
$$

We suppose that $\mathbf{f}$ is differentiable and has locally Lipschitz continuous first derivatives with respect to pose and perturbation, e.g., $\mathbf{f}$ is twice differentiable with respect to these variables. The Jacobian matrix of $\mathbf{f}$ with respect to variables $\mathbf{x}$ and $\mathbf{p}$ are denoted respectively as $\mathbf{F}_{\mathbf{x}}(\mathbf{x}, \mathbf{q}, \mathbf{p})$, called the kinematic parallel Jacobian matrix, and $\mathbf{F}_{\mathbf{p}}(\mathbf{x}, \mathbf{q}, \mathbf{p})$, called the sensitivity Jacobian matrix.

The nominal generalized workspace is defined by

$$
\mathcal{G}:=\left\{(\mathbf{x}, \mathbf{q}) \in \mathbb{R}^{n} \times \mathbb{R}^{n^{\prime}}: \mathbf{f}(\mathbf{x}, \mathbf{q})=\mathbf{0} \wedge \boldsymbol{g}(\mathbf{x}, \mathbf{q}) \leq \mathbf{0}\right\}
$$

where $\boldsymbol{g}$ is a set of inequalities that defines the generalized workspace of interest. We assume that $\mathcal{G}$ is bounded. We also require that $\mathcal{G}$ does not contain any parallel singularity, but this will be checked by the proposed method. 
In some situations, the kinematic model can be solved for the pose coordinates inside $\mathcal{G}$, giving rise to a direct model $\mathbf{d}: \mathbb{R}^{n^{\prime}} \rightarrow \mathbb{R}^{n}$ that provides an explicit description of the nominal generalized workspace:

$$
\mathcal{G}=\left\{(\mathbf{x}, \mathbf{q}) \in \mathbb{R}^{n} \times \mathbb{R}^{n^{\prime}}: \mathbf{x}=\mathbf{d}(\mathbf{q}) \wedge g(\mathbf{x}, \mathbf{q}) \leq 0\right\} .
$$

When perturbations are to be taken into account, we expect to have a direct model that depends of perturbations: $\mathbf{x}=\mathbf{d}(\mathbf{q}, \mathbf{p})$. Such a direct model is generally not correct for arbitrarily large perturbations, and therefore has to be associated to a perturbation domain $\mathcal{P}$ for which it is valid (for simplicity, the perturbation domain $\mathcal{P}$ is supposed to contain $\mathbf{0}$ and to be convex). Although such a direct model with explicit dependence on perturbations naturally arises in the context of serial robots, it is usually quite difficult to obtain for parallel robots, or even does not exists.

The pose error is the error between the nominal pose and the perturbed pose for fixed actuated joint coordinates. When a direct kinematic model is available, this can be expressed as $e(\mathbf{d}(\mathbf{q}, \mathbf{0}), \mathbf{d}(\mathbf{q}, \mathbf{p}))$, where $e$ either the norm of the positioning error or the norm of the orientation error, so $e\left(\mathbf{x}, \mathbf{x}^{\prime}\right)=\left\|\mathbf{\Pi}\left(\mathbf{x}-\mathbf{x}^{\prime}\right)\right\|$ where $\Pi$ is a projection on a subset of the coordinates of the pose. When no direct model is available, the situation is more complex since it becomes difficult to associate perturbed poses to their nominal counterpart. This is investigated in the next section.

\section{Computing a sharp upper bound for maximal pose errors (Step 3)}

When an explicit direct model $\mathbf{x}=\mathbf{d}(\mathbf{q}, \mathbf{p})$ is available, e.g., in the case of a serial robot, finding the maximal pose error over the workspace can be modeled by the following constrained optimization problem:

$$
\max _{\substack{(\mathbf{x}, \mathbf{q}) \in \mathcal{G} \\ \mathbf{p} \in \mathcal{P}}} e\left(\mathbf{x}, \mathbf{x}^{\prime}\right),
$$

see e.g. [19]. The variables are the joint coordinates $\mathbf{q} \in \mathbb{R}^{n^{\prime}}$, the nominal pose $\mathbf{x} \in \mathbb{R}^{n}$, the perturbed pose $\mathbf{x}^{\prime} \in \mathbb{R}^{n}$ and the perturbation $\mathbf{p} \in \mathbb{R}^{m}$. The constraint $(\mathbf{x}, \mathbf{q}) \in \mathcal{G}$ states that $(\mathbf{x}, \mathbf{q})$ is a nominal configuration, while the constraint $\mathbf{x}^{\prime}=\mathbf{d}(\mathbf{q}, \mathbf{p})$ states that $\mathbf{x}^{\prime}$ is the perturbed pose corresponding to the same joint coordinates $\mathbf{q}$.

The aim of this section is to generalize this approach to the case where the direct model with explicit dependence on perturbations is not available. The naive generalization

$$
\max _{\substack{(\mathbf{x}, \mathbf{q}) \in \mathcal{G} \\ \mathbf{p} \in \mathcal{P} \\ \mathbf{f}\left(\mathbf{x}^{\prime}, \mathbf{q}, \mathbf{p}\right)=\mathbf{0}}} e\left(\mathbf{x}, \mathbf{x}^{\prime}\right)
$$

raises two issues: First, it is difficult to define a domain for the perturbed pose $\mathbf{x}^{\prime}$ unless we know an upper bound for the pose error a priori. Second, (8) 

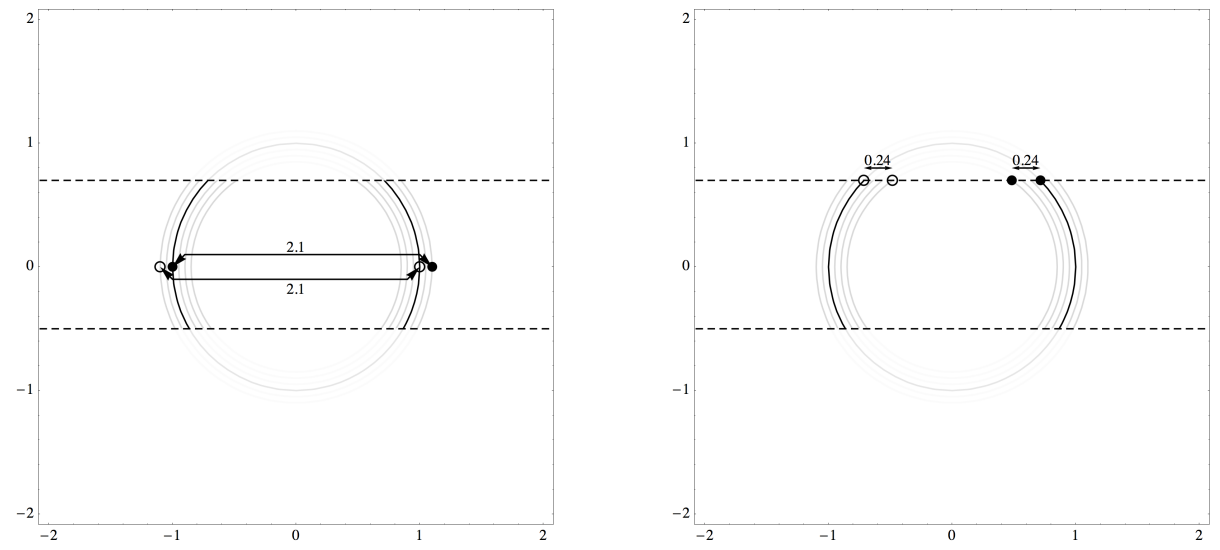

Figure 1: In black the nominal solutions of Example2 in gray its perturbed solutions. Left: Solutions of Problem (9). Right: Solutions of Problem [13).

is actually not correct because the direct kinematic problem may have several solutions or no solution at all for a given perturbation. This means that the maximum of $e\left(\mathbf{x}, \mathbf{x}^{\prime}\right)$ is likely to be reached for a $\mathbf{x}^{\prime}$ that does not corresponds to a perturbation of $\mathbf{x}$, i.e., the optimization problem (8) makes no sense in genera 1 . The following example illustrates this situation.

Example 2. Consider the equation $x^{2}+q^{2}=(1+p)^{2}$, and the domains $x \in$ $[-10,10], q \in[-0.5,0.7]$ and $\mathcal{P}=[-0.1,0.1]$. For each $q \in \mathbf{q}$ and $p \in \mathcal{P}$, the system has two solutions $x= \pm \sqrt{(1+p)^{2}-q^{2}}$ (see Figure 1). In this simple case, perturbed solutions are easily related to their nominal solution: positive (respectively negative) perturbed solutions correspond to the positive (respectively negative) nominal solution. The pose error maximization problem (8) is

$$
\begin{gathered}
\max _{x \in[-10,10], q \in[-0.5,0.7]}\left|x-x^{\prime}\right| . \\
x^{2}+q^{2}=1 \\
p \in[-0.1,0.1] \\
x^{\prime 2}+q^{2}=(1+p)^{2}
\end{gathered}
$$

This simple maximization problem can be solved formally and the maximum shown to be 2.1. This correspond to the worst distance between the negative nominal solution and a positive perturbed solution (black disks on the left graphic of Figure 1), or vice versa (black circles on the left graphic of Figure 1). Although it is an upper bound for the pose error, it is so much overestimated, since

\footnotetext{
${ }^{1}$ Often, workspaces are designed so that the direct kinematic problem has only one solution in the workspace. However, uncertainties may introduce other solutions, and it turns out to be difficult to certify they actually do not (such a certification is provided by the methodology proposed in the present paper). Furthermore, this may not always be the case, e.g. for cuspidal robots 20, 21] or when singularities are allowed to be crossed for enlarging the workspace 22].
} 
perturbed solutions are compared to the two nominal solutions instead of being compared only to its associated nominal solution, that it makes no senses.

Hence, the perturbed pose has to be somehow related only to its corresponding nominal solution. We formalize this restriction as follows: For given $\bar{\epsilon}>0$, $\mathcal{G} \subseteq \mathbb{R}^{n} \times \mathbb{R}^{n}$ and $\mathcal{P} \subseteq \mathbb{R}^{m}$, we say that $\mathcal{G}$ is $\bar{\epsilon}$-safe with respect to $\mathcal{P}$ if

$$
\forall(\mathbf{x}, \mathbf{q}) \in \mathcal{G}, \forall \mathbf{p} \in \mathcal{P}, \exists ! \mathbf{x}^{\prime} \in \bar{B}(\mathbf{x}, \bar{\epsilon}), \mathbf{f}\left(\mathbf{x}^{\prime}, \mathbf{q}, \mathbf{p}\right)=\mathbf{0} .
$$

The existence of a unique perturbed pose within $\bar{B}(\mathbf{x}, \bar{\epsilon})$ is crucial in this definition since it enforces a functional dependence between $\mathbf{x}^{\prime}$ and $\mathbf{p} \in \mathcal{P}$, which we denote by $\mathbf{x}^{\prime}=\mathbf{d}_{\mathbf{x}, \mathbf{q}}(\mathbf{p})$. This function is continuous $2^{2}$, and $\mathbf{d}_{\mathbf{x}, \mathbf{q}}(\mathbf{0})=\mathbf{x}$, which makes $\mathbf{x}^{\prime}=\mathbf{d}_{\mathbf{x}, \mathbf{q}}(\mathbf{p})$ the perturbed pose associated to the nominal pose $\mathbf{x}$ with no ambiguity. Therefore, under the hypothesis that $\mathcal{G}$ is $\bar{\epsilon}$-safe with respect to $\mathcal{P}$, we can safely use the kinematic model $\mathbf{f}\left(\mathbf{x}^{\prime}, \mathbf{q}, \mathbf{p}\right)=\mathbf{0}$ in order to characterize the perturbed pose:

$$
\begin{gathered}
(\mathbf{x}, \mathbf{q}) \in \mathcal{G}, \mathbf{p} \in \mathcal{P} \\
\mathbf{x}^{\prime} \in \bar{B}(\mathbf{x}, \bar{\epsilon}) \\
\mathbf{f}\left(\mathbf{x}^{\prime}, \mathbf{q}, \mathbf{p}\right)=\mathbf{0}
\end{gathered} \quad \Longleftrightarrow \quad \begin{gathered}
(\mathbf{x}, \mathbf{q}) \in \mathcal{G}, \mathbf{p} \in \mathcal{P} \\
\mathbf{x}^{\prime}=\mathbf{d}_{\mathbf{x}, \mathbf{q}}(\mathbf{p})
\end{gathered}
$$

As a consequence, even though no explicit expression of $\mathbf{d}_{\mathbf{x}, \mathbf{q}}$ is available, the maximal error inside the nominal workspace is given by

$$
\begin{gathered}
\max _{\substack{\mathbf{x}, \mathbf{q}) \in \mathcal{G} \\
\mathbf{p} \in \mathcal{P}}} e\left(\mathbf{x}, \mathbf{x}^{\prime}\right) . \\
\mathbf{x}^{\prime} \in \bar{B}(\mathbf{x}, \bar{\epsilon}) \\
\mathbf{f}\left(\mathbf{x}^{\prime}, \mathbf{q}, \mathbf{p}\right)=\mathbf{0}
\end{gathered}
$$

Example 3. For the same problem as in Example 2, $\bar{\epsilon}=0.3$ is an upper bound for the pose error. Then the pose error maximization problem 12 is

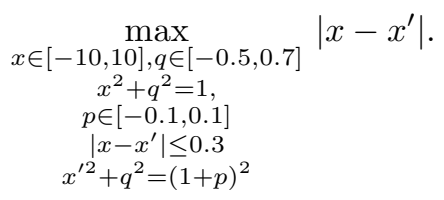

This problem can also be solved formally, leading to a maximal pose error of 0.23196. This now corresponds to the exact pose error upper bound shown on the right graphic of Figure 1 .

As a conclusion, the constrained optimization problem (12) allows computing a sharp pose error upper bound, provided that the nominal generalized workspace $\mathcal{G}$ is proved to be $\bar{\epsilon}$-safe with respect to $\mathcal{P}$. The next subsection deals with the determination of $\mathcal{P}$ and $\bar{\epsilon}$ using Kantorovich theorem.

\footnotetext{
${ }^{2}$ Indeed, if it was not continuous at some $\mathbf{p}_{\infty} \in \mathcal{P}$ then there would exist a sequence $\mathbf{p}_{k} \in \mathcal{P}$ converging to $\mathbf{p}_{\infty}$, such that $\mathbf{x}_{k}:=\mathbf{d}_{\mathbf{x}, \mathbf{q}}\left(\mathbf{p}_{k}\right)$ does not converge to $\mathbf{x}_{\infty}:=\mathbf{d}_{\mathbf{x}, \mathbf{q}}\left(\mathbf{p}_{\infty}\right)$. Since, the sequence $\mathbf{x}_{k}$ is bounded, this implies that it has an accumulation point $\mathbf{x}^{*}$ different of $\mathbf{x}_{\infty}$. However, by continuity of $\mathbf{f}$, we have $\mathbf{f}\left(\mathbf{x}^{*}, \mathbf{q}, \mathbf{p}_{\infty}\right)=\mathbf{0}$ and the perturbation $\mathbf{p}_{\infty}$ gives rise to two different perturbed poses $\mathbf{x}^{*}$ and $\mathbf{x}_{\infty}$ inside $\bar{B}(\mathbf{x}, \bar{\epsilon})$, a contradiction.
} 
Remark 1. The error function in $(12)$ can be chosen to be either the norm of the position error or the norm of the orientation error, which is mandatory since aggregating these errors is often difficult or irrelevant. However, both position and orientation errors are aggregated in the norm condition $\mathbf{x}^{\prime} \in \bar{B}(\mathbf{x}, \bar{\epsilon})$ of (10), (11) and (12). Although lacking physical meaning, this aggregation is necessary to obtain a first crude upper bound. The impact of this aggregation is on the size of the perturbation domain $\mathcal{P}$.

\section{Parametric Kantorovich theorem for $\bar{\epsilon}$-safety (Step 1)}

In this section, we show how Kantorovich theorem can be used in a parametric way within the whole generalized workspace and perturbation space in order to provide both a perturbation domain for which the above uniqueness condition holds, and a pose error upper bound valid within the whole workspace and for all considered perturbations.

\subsection{Informal presentation}

Kantorovich theorem 22, 24, 15, 25, (see Appendix Appendix A) is applied in order to both provide a perturbation domain $\mathcal{P}$ and an error upper bound $\bar{\epsilon}$ for which $\mathcal{G}$ is $\bar{\epsilon}$-safe with respect to $\mathcal{P}$.

The basic idea is, for an arbitrary nominal configuration $\left(\mathbf{x}_{0}, \mathbf{q}\right) \in \mathcal{G}$ and an arbitrary perturbation $\mathbf{p} \in \mathcal{P}$, to apply Kantorovich theorem for solving the perturbed direct kinematic problem $\mathbf{h}(\mathbf{x}):=\mathbf{f}(\mathbf{x}, \mathbf{q}, \mathbf{p})=\mathbf{0}$, with the nominal pose $\mathbf{x}_{0}$ as an initial condition. Both $\mathbf{q}$ and $\mathbf{p}$ are thus considered as parameters of a square system of equations in $\mathbf{x}$. They are however handled in two different ways: The constants of Kantorovich theorem are maximized with respect to $\mathbf{q}$, leading to worst case existence and uniqueness domains over the whole generalized workspace. The dependance of Kantorovich constants w.r.t. $\mathbf{p}$ is more accurately handled by computing second order derivatives with w.r.t. p, hence providing existence and uniqueness domains that explicitly depend on $\mathbf{p}$. The key feature of Kantorovich theorem that allows applying it over the whole generalized workspace and perturbation space is that when the constants involved in Kantorovich theorem are sharper, the size of the existence ball decreases and the size of the uniqueness ball increases. Therefore, worst case constants over $\mathcal{G}$ and $\mathcal{P}$ will provide a greatest existence domain and a smallest uniqueness domain valid over both $\mathcal{G}$ and $\mathcal{P}$.

Finally, the perturbation domain $\mathcal{P}$ is going to be defined so that the hypotheses of Kantorovich theorem are satisfied for every perturbation it contains. As a consequence, the existence and uniqueness regions provided by Kantorovich theorem are going to enforce $\mathcal{G}$ to be $\bar{\epsilon}$-safe with respect to $\mathcal{P}$, for a given $\bar{\epsilon}$ also provided by Kantorovich theorem.

Remark 2. The interval Newton also allows performing a parametric existence proof (see e.g. [26, 27] where parallelotopes where used to enclose one manifolds, and [28, 29] where boxes where used to enclose higher dimensional manifolds, in particular in the context of computing generalized workspaces), and could be 
used here. Although the interval Newton has been shown to be more efficient in terms of proving power in [30], its usage in the context of parametric error bound estimation would require to apply it everywhere on the generalized workspace. On the other hand, the parametric usage of Kantorovich theorem presented in the next subsection only requires that some upper bounds are computed over the generalized workspace, which is much less time consuming since branch and bound algorithms do not explore the whole feasible set for computing such upper bounds.

\subsection{The main theorem}

Choose an apriori maximal tolerance $\bar{\Delta}>0$ so that only perturbations satisfying $\|\mathbf{p}\| \in \bar{B}_{\bar{\Delta}}$ will be considered ${ }^{3}$. Then define the constants $\kappa, \chi$ and $\gamma^{(i)}$ such that:

$$
\begin{aligned}
\kappa & \geq \max _{\substack{(\mathbf{x}, \mathbf{q}) \in \mathcal{G} \\
\mathbf{p} \in \bar{B}_{\bar{\Delta}}}}\|\mathbf{f}(\mathbf{x}, \mathbf{q}, \mathbf{p})\| \\
\chi & \geq \max _{\substack{(\mathbf{x}, \mathbf{q}) \in \mathcal{G} \\
\mathbf{p} \in \bar{B}_{\bar{\Delta}}}}\left\|\mathbf{F}_{\mathbf{x}}(\mathbf{x}, \mathbf{q}, \mathbf{p})^{-1}\right\| \\
\gamma^{(i)} & \geq \max _{\substack{(\mathbf{x}, \mathbf{q}) \in \mathcal{G} \\
\mathbf{p} \in \bar{B}_{\bar{\Delta}}}}\left\|\mathbf{F}_{\mathbf{x}}(\mathbf{x}, \mathbf{q}, \mathbf{p})^{-1} \mathbf{F}_{\mathbf{p}^{(i)}}(\mathbf{x}, \mathbf{q}, \mathbf{0})\right\|
\end{aligned}
$$

where $\mathbf{p}^{(i)}$ form a partition of the perturbation vector $\mathbf{p}$. This partition will allow to do a separate tolerance synthesis for each type of parameters, see examples in Section 6. Define

$$
r:=\kappa \chi,
$$

and suppose the two following Lipschitz conditions hold for constants $\lambda$ and $\mu$ :

$$
\begin{aligned}
\forall\left(\mathbf{x}_{0}, \mathbf{q}\right) \in \mathcal{G}, \forall \mathbf{p} \in \bar{B} \bar{\Delta}, \forall \mathbf{x}^{\prime}, \mathbf{x}^{\prime \prime} \in \bar{B}\left(\mathbf{x}_{0},(2 r)^{+}\right), \\
\left\|\mathbf{F}_{\mathbf{x}}\left(\mathbf{x}^{\prime}, \mathbf{q}, \mathbf{p}\right)-\mathbf{F}_{\mathbf{x}}\left(\mathbf{x}^{\prime \prime}, \mathbf{q}, \mathbf{p}\right)\right\| \leq \lambda\left\|\mathbf{x}^{\prime}-\mathbf{x}^{\prime \prime}\right\|,
\end{aligned}
$$

where $(2 r)^{+}$is strictly greater than $2 r$ (in practice we choose $(2 r)^{+}$as the smallest representable floating point number greater that $2 r$ ), and

$$
\forall(\mathbf{x}, \mathbf{q}) \in \mathcal{G}, \forall \mathbf{p}, \mathbf{p}^{\prime} \in \bar{B}_{\bar{\Delta}},\left\|\mathbf{F}_{\mathbf{p}}(\mathbf{x}, \mathbf{q}, \mathbf{p})-\mathbf{F}_{\mathbf{p}}\left(\mathbf{x}, \mathbf{q}, \mathbf{p}^{\prime}\right)\right\| \leq \mu\left\|\mathbf{p}-\mathbf{p}^{\prime}\right\| .
$$

Theorem 1. Let $\bar{\Delta}>0$, and $\kappa, \chi, \gamma \geq 0$ be such that 14, 15) and (16) are satisfied, and define $r:=\kappa \chi$ as in (17). Consider $\lambda, \mu \geq 0$ such that 18 and (19) are satisfied. Define furthermore

$$
\eta(\mathbf{p}):=\sum_{i} \gamma^{(i)}\left\|\mathbf{p}^{(i)}\right\|+\frac{1}{2} \mu \chi\|\mathbf{p}\|^{2}
$$

\footnotetext{
${ }^{3}$ Fixing the value of $\bar{\Delta}$ can be done with successive adjustments when necessary: Too large or too small values of $\bar{\Delta}$ will result in small perturbation domain, see Footnote 8 page 14
} 
and the perturbation domain

$$
\mathcal{P}:=\left\{\mathbf{p} \in \bar{B}_{\bar{\Delta}}: 2 \lambda \chi \eta(\mathbf{p}) \leq 1\right\} .
$$

Then $\mathcal{G}$ is $\bar{\epsilon}$-safe with respect to $\mathcal{P}$ for

$$
\bar{\epsilon}:=\min \left\{2 r, \frac{1}{\chi^{\lambda}}\right\} .
$$

Furthermore, the distance between the nominal and the perturbed poses is at most

$$
\epsilon(\mathbf{p}):=\frac{2 \eta(\mathbf{p})}{1+\sqrt{1-2 \chi \lambda \eta(\mathbf{p})}} .
$$

Preliminary Remarks. Both $r$ and $\eta(\mathbf{p})$ are going to be proved to be upper bounds of the (norm of the) first Newton step performed for solving the perturbed system starting at a nominal solution. The first is crude because independent of $\mathbf{p}$, and will used mostly to define the domain where to evaluate the Lipschitz constant $\lambda$ in (18). The second bounds the first Newton step accurately w.r.t. $\mathbf{p}$.

The perturbation domain $\mathcal{P}$ is then defined exactly so that the hypothesis $2 \chi \lambda \delta \leq 1$ of Kantorovich theorem is satisfied. Therefore, Kantorovich theorem will naturally apply to these perturbations. The existence domain will be sharply depending on the perturbation, while the uniqueness domain will be crude and independent of the parameters, though large enough to be used in Step 3. Finally, the sharp pose error upper bound $\epsilon(\mathbf{p})$ is just $t^{*}(\chi, \lambda, \eta(\mathbf{p}))$, i.e., the radius of the existence domain provided by Kantorovich theorem.

Proof. Consider an arbitrary nominal pose $\left(\mathbf{x}_{0}, \mathbf{q}\right) \in \mathcal{G}$. We just need to prove that for an arbitrary fixed $\mathbf{p} \in \mathcal{P}$ there exists a unique solution $\mathbf{x}^{*}$ to $\mathbf{h}(\mathbf{x})=\mathbf{0}$, with $\mathbf{h}(\mathbf{x}):=\mathbf{f}(\mathbf{x}, \mathbf{q}, \mathbf{p})$, inside $\bar{B}\left(\mathbf{x}_{0}, \bar{\epsilon}\right)$, and that this solution satisfies $\left\|\mathbf{x}_{0}-\mathbf{x}^{*}\right\| \leq \epsilon(\mathbf{p})$.

To this end, we apply Corollary 1 to the system $\mathbf{h}(\mathbf{x})=\mathbf{0}$, starting from the initial guess $\mathbf{x}_{0}$. By 15$], \boldsymbol{\Gamma}_{0}:=\mathbf{H}_{\mathbf{x}}\left(\mathbf{x}_{0}\right)^{-1}$ is defined and

$$
\left\|\boldsymbol{\Gamma}_{0}\right\| \leq \chi
$$

Define

$$
\delta(\mathbf{p}):=\min \{r, \eta(\mathbf{p})\}
$$

We prove now that

$$
\left\|\boldsymbol{\Gamma}_{0} \mathbf{h}\left(\mathbf{x}_{0}\right)\right\| \leq \delta(\mathbf{p}) .
$$

First, $\left\|\boldsymbol{\Gamma}_{0} \mathbf{h}\left(\mathbf{x}_{0}\right)\right\| \leq\left\|\boldsymbol{\Gamma}_{0}\right\|\left\|\mathbf{h}\left(\mathbf{x}_{0}\right)\right\| \leq \kappa \chi=r$, the second inequality resulting of 14) and 15). Second, defining $\mathbf{M}:=\mathbf{F}_{\mathbf{p}}\left(\mathbf{x}_{0}, \mathbf{q}, \mathbf{0}\right), \mathbf{M}^{(i)}:=\mathbf{F}_{\mathbf{p}^{(i)}}\left(\mathbf{x}_{0}, \mathbf{q}, \mathbf{0}\right)$ 
and $\mathbf{z}:=\mathbf{h}\left(\mathbf{x}_{0}\right)-\mathbf{M p}$,

$$
\begin{aligned}
\left\|\boldsymbol{\Gamma}_{0} \mathbf{h}\left(\mathbf{x}_{0}\right)\right\| & =\left\|\boldsymbol{\Gamma}_{0}(\mathbf{M} \mathbf{p}+\mathbf{z})\right\| \\
& =\left\|\boldsymbol{\Gamma}_{0}\left(\sum_{i} \mathbf{M}^{(i)} \mathbf{p}^{(i)}+\mathbf{z}\right)\right\| \\
& \leq \sum_{i}\left\|\boldsymbol{\Gamma}_{0} \mathbf{M}^{(i)}\right\|\left\|\mathbf{p}^{(i)}\right\|+\left\|\boldsymbol{\Gamma}_{0}\right\|\|\mathbf{z}\| \\
& \leq \sum_{i} \gamma^{(i)}\left\|\mathbf{p}^{(i)}\right\|+\chi\|\mathbf{z}\|,
\end{aligned}
$$

where the last inequality follows from $(15)$ and $(16)$. Finally, following the classical argument given in [23, 25] and using (19), we have $\|\mathbf{z}\| \leq \frac{1}{2} \mu\|\mathbf{p}\|^{2}$, which proves (26).

Second, We have $r \geq \delta(\mathbf{p})$ by the definition (25) of $\delta(\mathbf{p})$, so $D_{0}:=B\left(\mathbf{x}_{0},(2 r)^{+}\right)$ satisfies Corollary 1 hypothesis. The Lipschitz condition of Corollary 1 is valid inside $D_{0}$ by (18). Finally,

$$
h=2 \chi \lambda \delta(\mathbf{p}) \underset{(25)}{\leq} 2 \lambda \chi \eta(\mathbf{p}) \underset{(21)}{\leq} 1 .
$$

Therefore all hypothesis of Corollary 1 are verified, and there exists a solution of $\mathbf{h}(\mathbf{x})=0$ inside

$$
\bar{B}\left(\mathbf{x}_{0}, t^{*}(\chi, \lambda, \delta(\mathbf{p}))\right) \subseteq \bar{B}\left(\mathbf{x}_{0}, t^{*}(\chi, \lambda, \eta(\mathbf{p}))\right)=\bar{B}\left(\mathbf{x}_{0}, \epsilon(\mathbf{p})\right),
$$

the first inclusion holding by (25) and Lemma 1 , the second equality holding by definition of $t^{*}$ and $\epsilon(\mathbf{p})$. This solution is unique inside $\bar{B}\left(\mathbf{x}_{0}, \min \left\{2 r, \frac{1}{\chi^{\lambda}}\right\}\right)=$ $\bar{B}\left(\mathbf{x}_{0}, \bar{\epsilon}\right)$.

Remark 3. The perturbation domain $\mathcal{P}$ provided by Theorem 1 is convex. Indeed, it has the form $\mathbf{a}^{T} \mathbf{p}+b\|\mathbf{p}\|^{2} \leq 1$, with $b>0$, so $\mathbf{a}^{T} \mathbf{p}+b\|\mathbf{p}\|^{2}$ is the sum of two convex functions.

Remark 4. Kantorovich theorem also proves that for every perturbation in the interior of $\mathcal{P}$, the perturbed pose is not a parallel singularity, which can be of great practical interes 5 . This property is not included in the statement of the

\footnotetext{
${ }^{4}$ Defining $\boldsymbol{g}(t)=\mathbf{f}\left(\mathbf{x}_{0}, \mathbf{q}, t \mathbf{p}\right)$ we have $\boldsymbol{g}(0)=\mathbf{f}\left(\mathbf{x}_{0}, \mathbf{q}, \mathbf{0}\right)=\mathbf{0}, \boldsymbol{g}(1)=\mathbf{f}\left(\mathbf{x}_{0}, \mathbf{q}, \mathbf{p}\right)=\mathbf{h}\left(\mathbf{x}_{0}\right)$, and therefore $\mathbf{z}=g(1)-\mathbf{F}_{\mathbf{p}}\left(x_{0}, \mathbf{q}, \mathbf{0}\right) \mathbf{p}$. Then $g(1)=g(0)+\int_{0}^{1} g^{\prime}(t) d t=\int_{0}^{1} \mathbf{F}_{\mathbf{p}}\left(x_{0}, \mathbf{q}, t \mathbf{p}\right) \mathbf{p} d t$. Now using $\left[19, \mathbf{F}_{\mathbf{p}}\left(x_{0}, \mathbf{q}, t \mathbf{p}\right) \in \mathbf{F}_{\mathbf{p}}\left(x_{0}, \mathbf{q}, \mathbf{0}\right)+t \mu\|\mathbf{p}\|[-1,1]\right.$, leading to $g(1) \in \mathbf{F}_{\mathbf{p}}\left(x_{0}, \mathbf{q}, \mathbf{0}\right) \mathbf{p}+$ $\frac{1}{2} \mu\|\mathbf{p}\|[-1,1] \mathbf{p}$, and finally $\left\|g(1)-\mathbf{F}_{\mathbf{p}}\left(x_{0}, \mathbf{q}, \mathbf{0}\right) \mathbf{p}\right\| \leq \frac{1}{2} \mu\|\mathbf{p}\|^{2}$.

${ }^{5}$ For perturbation on the boundary of $\mathcal{P}$, we have $2 \lambda \chi \delta=1$ in Kantorovich theorem, which nevertheless proves the existence and uniqueness, but fail at proving the non-singularity. Indeed, consider the equation $f(x)=x^{2}=0$ with a positive initial condition $x_{0}$. In this case, $\chi=\frac{1}{2 x_{0}}, \delta=\frac{x_{0}}{1}$ and $\lambda=2$. Therefore $2 \lambda \chi \delta=1$, and the Newton iteration indeed converges toward the singular solution $x=0$.
} 
theorem, but is used in its proof. It can be summarized as follows: Let $\mathbf{x}_{\mathbf{p}}$ be the unique perturbed pose associated to the nominal pose $\mathbf{x}_{0}$. Then,

$$
\begin{array}{ccc} 
& & \left\|\mathbf{H}_{\mathbf{x}}\left(\mathbf{x}_{0}\right)^{-1} \mathbf{H}_{\mathbf{x}}\left(\mathbf{x}_{\mathbf{p}}\right)-I\right\| \\
= & & \left\|\mathbf{H}_{\mathbf{x}}\left(\mathbf{x}_{0}\right)^{-1}\left(\mathbf{H}_{\mathbf{x}}\left(\mathbf{x}_{\mathbf{p}}\right)-\mathbf{H}_{\mathbf{x}}\left(\mathbf{x}_{0}\right)\right)\right\| \\
\leq & \chi \lambda\left\|\mathbf{x}_{\mathbf{p}}-\mathbf{x}_{0}\right\| \\
\leq & \chi \lambda \epsilon(\mathbf{p}) \\
\leq & 2 \chi \lambda \eta(\mathbf{p}) .
\end{array}
$$

Now, for $\mathbf{p} \in \operatorname{int} \mathcal{P}$ we have $2 \chi \lambda \eta(\mathbf{p})<1$, which proves that $\mathbf{H}_{\mathbf{x}}\left(\mathbf{x}_{0}\right)^{-1} \mathbf{H}_{\mathbf{x}}\left(\mathbf{x}_{\mathbf{p}}\right)$ is nonsingula ${ }^{6}$, and hence $\mathbf{H}_{\mathbf{x}}\left(\mathbf{x}_{\mathbf{p}}\right)$ is also nonsingular.

If the absence of parallel singularities is to be proved then one can synthesize tolerances inside a slightly smaller perturbation domain, e.g., $\left\{\mathbf{p} \in \bar{B}_{\bar{\Delta}}\right.$ : $2 \lambda \chi \eta(\mathbf{p}) \leq 0.99999\}$ with no practical impact, but enforcing the perturbed pose not to be a parallel singularity.

Remark 5. An asymptotic analysis for small perturbations brings interesting insights: It can be seen from the definition (20) that

$$
\eta(\mathbf{p}) \approx \sum_{i} \gamma^{(i)}\left\|\mathbf{p}^{(i)}\right\|
$$

provided that

$$
\|\mathbf{p}\|^{2} \ll \frac{2}{\mu \chi} \sum_{i} \gamma^{(i)}\left\|\mathbf{p}^{(i)}\right\|,
$$

which is satisfied as soon as $\|\mathbf{p}\|$ is small enough. If furthermore $\chi \lambda \eta(\mathbf{p}) \approx$ $2 \chi \lambda \sum_{i} \gamma^{(i)}\left\|\mathbf{p}^{(i)}\right\|$ is small with respect to 1 then $\epsilon(\mathbf{p}) \approx \eta(\mathbf{p})=\sum_{i} \gamma^{(i)}\left\|\mathbf{p}^{(i)}\right\|$.

Another interesting observation is that, under the validity of the approximation $\eta(\mathbf{p}) \approx \sum_{i} \gamma^{(i)}\left\|\mathbf{p}^{(i)}\right\|$, the size of the perturbation domain $\left\{\mathbf{p} \in \bar{B}_{\bar{\Delta}}\right.$ : $\lambda \chi \eta(\mathbf{p}) \leq 1\}$ is proportional to the inverse of $\chi, \lambda$ and $\gamma^{(i)}$.

\subsection{An academic example: The $P R R P$ robot}

In order to illustrate the usage of Theorem 1 , we consider a simple academic one degree of freedom robot, the PRRP robot [29, represented in Figure 2a Obviously, the kinematic model of this robot take the following form:

$$
(x-a)^{2}+(q-b)^{2}=l^{2},
$$

whose solution set is represented in Figure 2b (serial and parallel singularities are represented by circles and black disks respectively). The geometric parameters value are supposed to be $a=1, b=1$ and $l=3$, and the nominal generalized workspace is expressed as:

$$
\mathcal{G}=\left\{(x, q) \in \mathbb{R}^{2}: 40 \wedge \wedge \leq x \leq 3 \wedge 3 \leq q \leq 4\right\},
$$

\footnotetext{
${ }^{6}$ It is well known that $\|M-I\|<1$ implies $M$ nonsingular, which can be seen, e.g., using Neumann series.
} 


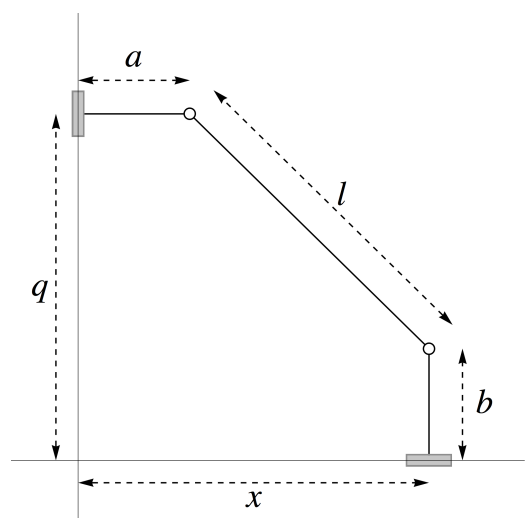

(a) Robot representation

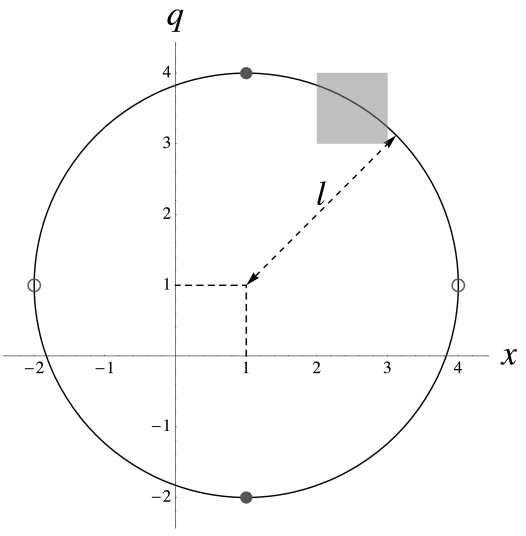

(b) Nominal generalized workspace

Figure 2: The $\underline{\text { RRP }}$ robot.

which is the intersection of the solution set of 40 and the gray rectangle depicted on Figure 2b.

We consider three perturbations $\mathbf{p}=\left(p_{1}, p_{2}, p_{3}\right)$ which acts respectively on $a$, $b$ and $l$ (we consider no partition of the perturbations), and an apriori maximal tolerance $\bar{\Delta}=0.1$. The upper bounds $\kappa, \chi$ and $\gamma$ are computed by solving the optimization problems (14), (15) and (16):

$$
\begin{aligned}
& \kappa \geq \max _{\substack{(\mathbf{x}, \mathbf{q}) \in \mathcal{G} \\
\mathbf{p} \in \bar{B}_{\bar{\Delta}}}}\left|\left(x-a-p_{1}\right)^{2}+\left(q-b-p_{2}\right)^{2}-\left(l+p_{3}\right)^{2}\right| \\
& \chi \geq \max _{\substack{(\mathbf{x}, \mathbf{q}) \in \mathcal{G} \\
\mathbf{p} \in \bar{B}_{\bar{\Delta}}}} \frac{1}{2\left|x-a-p_{1}\right|} \\
& \gamma \geq \max _{\substack{(\mathbf{x}, \mathbf{q}) \in \mathcal{G} \\
\mathbf{p} \in \bar{B}_{\bar{\Delta}}}} \frac{\left|x-a-p_{1}\right|+\left|q-b-p_{2}\right|+\left|l+p_{3}\right|}{\left|x-a-p_{1}\right|} .
\end{aligned}
$$

The upper bounds $\kappa=1.48, \chi=0.56$ and $\gamma=7.75$ have been computed using the global solver IBEX 7 [31, 32, 33 in less than 0.1 second on a standard laptop for each of them. Since the kinematic model of the robot is quadratic with respect to its variables and perturbations, we can obtain the Lipschitz constants $\lambda=2$ and $\mu=2$ without solving the optimization problems (18) and 19 .

\footnotetext{
${ }^{7}$ Optimization problems to be solved are non-convex, non-smooth and equality and inequality constrained, and we require rigorous upper bounds. Up to our knowledge, IBEX, which implements interval based constraint programming within a standard branch and bound algorithm, is currently the only software available that is able to solve such problems.
} 


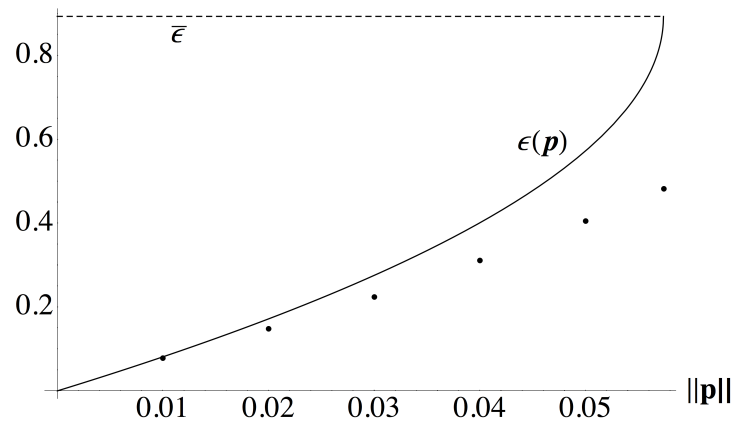

Figure 3: Upper bounds $\bar{\epsilon}$ and $\epsilon(\mathbf{p})$ resulting of the application of Theorem 1 as well as sharp upper bounds for the tolerances $\|\mathbf{p}\| \leq \Delta \in\{0.057,0.05,0.04,0.03,0.02,0.01\}$.

Applying Theorem 1, we obtain:

$$
\begin{aligned}
\eta(\mathbf{p}) & =15.5\|\mathbf{p}\|+1.12\|\mathbf{p}\|^{2} \\
\mathcal{P} & =\left\{\mathbf{p} \in \mathbb{R}^{3}:\|\mathbf{p}\| \leq 0.057\right\} \\
\bar{\epsilon} & =0.90, \\
\epsilon(\mathbf{p}) & =\frac{\eta(\mathbf{p})}{1+\sqrt{1-1.12 \eta(\mathbf{p})}}
\end{aligned}
$$

These results are depicted in Figure 3 . The perturbation domain for $\bar{\epsilon}$-safety is approximately $2 \%$ of $l$, which is realistic 8 .

We can now solve the optimization problem 12 , which provides a sharp upper bound of the end-effector pose error for $\|\mathbf{p}\| \leq \Delta \leq 0.057$ :

$$
\begin{gathered}
\max _{(x-a)^{2}+(q-b)^{2}=l^{2}}\left|x-x^{\prime}\right| . \\
\left(x^{\prime}-a-p_{1}\right)^{2}+\left(q-b-p_{2}\right)^{2}=\left(l-p_{3}\right)^{2} \\
2 \leq x \leq 3,3 \leq q \leq 4 \\
\|\mathbf{p}\| \leq \Delta \\
\left|x^{\prime}-x\right| \leq \bar{\epsilon}
\end{gathered}
$$

The variables of this optimization problem are $x, x^{\prime}, q$ and $\mathbf{p}$. The results obtained with IBEX for various values of $\Delta$ are computed in less than 0.1 second for each value of $\Delta$, and are displayed in Figure 3 using black dots. We can see that the actual error behaves approximately linearly with respect to $\|\mathbf{p}\|$, which motivates a linearization of the worst case pose error studied in the next section.

\footnotetext{
${ }^{8}$ Since $\max _{\mathbf{p} \in \mathcal{P}}\|\mathbf{p}\|=0.057<\bar{\Delta}=0.1$, we can enlarge $\mathcal{P}$ by decreasing $\bar{\Delta}$. E.g., fixing $\bar{\Delta}=0.07$ leads to $\left\{\mathbf{p} \in \mathbb{R}^{3}:\|\mathbf{p}\| \leq 0.062\right\}$.
} 


\section{Tolerance synthesis using linearization of the worst case pose error (Step 2)}

Let $\mathcal{P}$ be the perturbation domain provided by Theorem 1. Our aim is to determine a vector of tolerances $\boldsymbol{\Delta}=\left(\Delta_{i}\right)$ such that

$$
\mathcal{S}_{\Delta}:=\left\{\mathbf{p} \in \mathbb{R}^{m}:\left\|\mathbf{p}^{(i)}\right\| \leq \Delta_{i}\right\}
$$

is contained inside $\mathcal{P}$, and that the error $e\left(\mathbf{x}, \mathbf{x}^{\prime}\right)$ is less than a given threshold $\bar{e}$ for all perturbations in $\mathcal{S}_{\boldsymbol{\Delta}}$.

In Subsection 5.1, we propose a non-rigorous linear approximation of the maximal error in the workspace. In Subsection 5.2, we formulate the Tolerance synthesis as a multi-objective optimization problem, aiming to maximize the different tolerances $\Delta_{i}$. Finally, a certified upper bound for the pose error corresponding to the tolerances synthesized by this process is computed by solving the optimization problem described Section 3

\subsection{Approximate linearization of the pose error}

Theorem 1 involves several overestimations, which leads to an overestimated error upper bound for the considered perturbation domain. We make the as-

sumption that a linear approximation is going to be accurate within the perturbation domain provided by Theorem 1 .

$$
\mathbf{x}-\mathbf{x}^{\prime} \approx \mathbf{F}_{\mathbf{x}}(\mathbf{x}, \mathbf{q}, \mathbf{0})^{-1} \mathbf{F}_{\mathbf{p}}(\mathbf{x}, \mathbf{q}, \mathbf{0}) \mathbf{p} .
$$

We obtain an approximate workspace worst case error $e(\boldsymbol{\Delta})$ in the following way: Using (51), we obtain that (12) is approximately

$$
\max _{\substack{(\mathbf{x}, \mathbf{q}) \in \mathcal{G} \\ \mathbf{p} \in \mathcal{S}_{\Delta}}}\left\|\mathbf{\Pi}\left(\mathbf{x}-\mathbf{x}^{\prime}\right)\right\| \approx \max _{\substack{(\mathbf{x}, \mathbf{q}) \in \mathcal{G} \\ \mathbf{p} \in \mathcal{S}_{\Delta}}}\left\|\mathbf{\Pi} \mathbf{F}_{\mathbf{x}}(\mathbf{x}, \mathbf{q}, \mathbf{0})^{-1} \mathbf{F}_{\mathbf{p}}(\mathbf{x}, \mathbf{q}, \mathbf{0}) \mathbf{p}\right\|
$$

which we approximate by

$$
\max _{\substack{(\mathbf{x}, \mathbf{q}) \in \mathcal{G} \\ \mathbf{p} \in \mathcal{S}_{\Delta}}}\left\|\boldsymbol{\Pi} \mathbf{F}_{\mathbf{x}}(\mathbf{x}, \mathbf{q}, \mathbf{0})^{-1} \mathbf{F}_{\mathbf{p}}(\mathbf{x}, \mathbf{q}, \mathbf{0})\right\|\|\mathbf{p}\|
$$

We finally approximate 53 by

$$
\max _{\mathbf{p} \in \mathcal{S}_{\Delta}} \sum_{i} \gamma_{0}^{(i)}\left\|\mathbf{p}^{(i)}\right\|=\sum_{i} \gamma_{0}^{(i)} \Delta_{i}=: e(\boldsymbol{\Delta})
$$

where

$$
\gamma_{0}^{(i)}=\max _{(\mathbf{x}, \mathbf{q}) \in \mathcal{G}}\left\|\mathbf{\Pi} \mathbf{F}_{\mathbf{x}}(\mathbf{x}, \mathbf{q}, \mathbf{0})^{-1} \mathbf{F}_{\mathbf{p}^{(i)}}(\mathbf{x}, \mathbf{q}, \mathbf{0})\right\|
$$

The constants $\gamma_{0}^{(i)}$ can be computed using global optimization, resulting in a nonlinear problem similar but simpler than (16). Although it is difficult to assess the accuracy of this linear approximation in general, it turns out to be very accurate in the preliminary experiments presented in Section 6. 


\subsection{Approximate tolerance synthesis}

In order to be able to compute a rigorous pose error upper bound using Theorem (1), we need to choose $\Delta_{i}$ using (54) under the additional constraint that $\mathcal{S}_{\boldsymbol{\Delta}}$ is a subset of $\mathcal{P}$. Therefore, admissible tolerances $\boldsymbol{\Delta}$ satisfy

$$
\begin{aligned}
\sum_{i} \gamma_{0}^{(i)} \Delta_{i} & \leq \bar{e} \\
2 \sum_{i} \gamma^{(i)} \Delta_{i}+\mu \chi\|\Delta\|^{2} & \leq \frac{1}{\lambda \chi} \\
\|\boldsymbol{\Delta}\| & \leq \bar{\Delta},
\end{aligned}
$$

where (57) and (58) encode $\mathcal{S}_{\boldsymbol{\Delta}} \subseteq \mathcal{P}$ (the constraint (57) comes from (20) and (21), while (58) is the a priori maximal perturbation norm). As mentioned earlier, the domain (57) is convex, and so is the set of admissible tolerances defined by (56), (57) and (58). Finally, we want to select tolerances satisfying (56), (57) and (58) that maximizes each $\Delta_{i}$ in a multi-objective sense, i.e., that are Pareto optimal for these objectives. Since objectives and constraints are convex and almost linear, this multi-objective optimization problem can be easily solved using weighted-sum or $\epsilon$-constraint methods [34, 35].

\subsection{Rigorous validation of the approximate tolerance synthesis}

Finally, solving the optimization problem 12 provides a rigorous upper bound for the pose error over the workspace for the synthesized tolerances. Two cases arise: Either the rigorous upper bound $[12$ is close enough to the approximate one (54) so that the synthesized tolerances can be used, or the process can be repeated for neighbor tolerances in order to achieve better tolerances. In the experiments presented in the next section, the approximate upper bound (54) is very accurate. However, it is less accurate in the case of the academic example of Section 4.3 , where $\gamma_{0} \approx 6.82$ while the linear model corresponding to points in Figure 3 is $e(\Delta) \approx 8.52 \Delta$.

\section{Preliminary experiments}

In this section, we provide some first simulations to assess the usefulness of the proposed method. The upper bounds $\kappa, \chi, \gamma^{(i)}$ and $\gamma_{0}^{(i)}$ have been rigorously computed solving the optimization problems (14), 15, (16) and (55) using the global solver IBEX [31, 32, 33. It allows computing an arbitrarily sharp certified upper bound of these optimization problems, and we stopped the computations when the relative precision of the maximum is $1 \%$ (all presented upper bounds are therefore certified and accurate). All computations have been performed on an Intel i7 $2.80 \mathrm{GHz}$, the code has not been parallelized, and all timing are given in seconds (a 1 hour timeout has been enforced).

Two different robots are investigated: A RPRPR in Subsection 6.1 and a 3-RPR, with constant orientation in Subsection 6.2. We consider no joint 

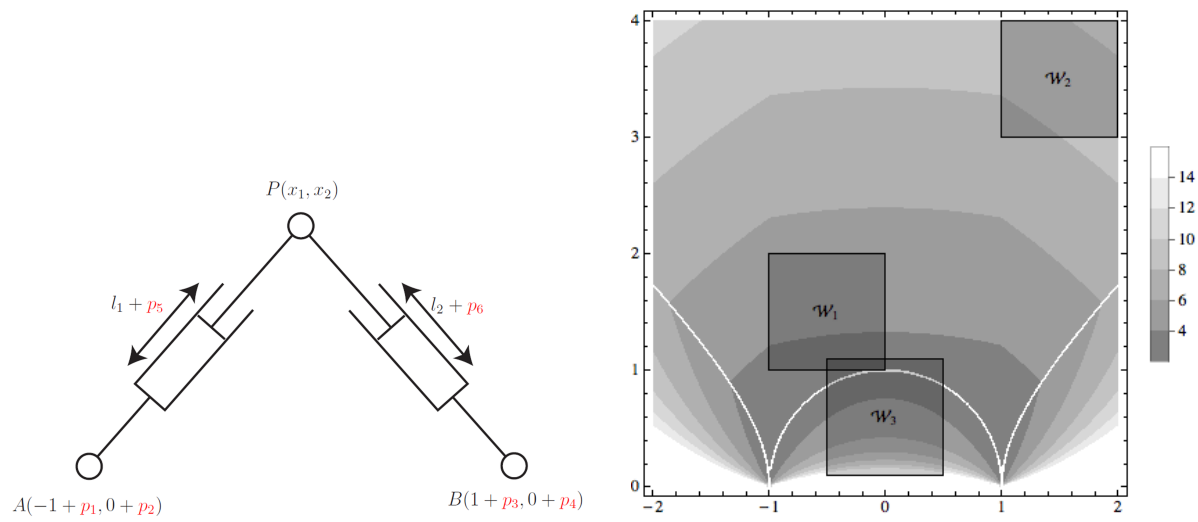

Figure 4: Left: The RPRPR robot. Right: Sensitivity index and the three workspaces considered for the R $\underline{P} \underline{P} R$ robot (white curves are drawing artefacts due to the non-differentiability of the sensitivity index).

limits, i.e. $q_{i} \in[0,+\infty)$. The generalized workspace of interest is defined by a rectangular domain in the cartesian space: $\mathbf{x} \in[\underline{\mathbf{x}}, \overline{\mathbf{x}}]=: \mathcal{W}$. Therefore,

$$
\mathcal{G}=\left\{(\mathbf{x}, \mathbf{q}) \in \mathbb{R}^{2 n}: \mathbf{f}(\mathbf{x}, \mathbf{q})=\mathbf{0}, \underline{\mathbf{x}} \leq \mathbf{x} \leq \overline{\mathbf{x}}\right\} .
$$

Several workspaces $\mathcal{W}_{i}$ are going to be investigated for each robot.

Remark 6. We formally invert the matrix $\mathbf{F}_{\mathbf{x}}(\mathbf{x}, \mathbf{q}, \mathbf{p})$ in order to solve 15 and (16). Tackling higher dimensional problems will require investigating some rigorous numerical inverse enclosure methods.

\subsection{The RPRPR robot}

In this subsection, we study the simple parallel robot RPRPR with 6 perturbations, 4 of them acting on the anchor points, and 2 of them are controlled, which is represented in the left graphic of Figure 4. Its kinematic model $\mathbf{F}(\mathbf{x}, \mathbf{q}, \mathbf{p})$ is therefore

$$
\begin{gathered}
\left(1+p_{1}+x_{1}\right)^{2}+\left(p_{2}+x_{2}\right)^{2}-\left(p_{3}-q_{1}\right)^{2} \\
\left(-1+p_{4}+x_{1}\right)^{2}+\left(p_{5}+x_{2}\right)^{2}-\left(p_{6}-q_{2}\right)^{2} .
\end{gathered}
$$

Since there is not orientation error, we use $e\left(\mathbf{x}, \mathbf{x}^{\prime}\right)=\left\|\mathbf{x}-\mathbf{x}^{\prime}\right\|$, i.e. $\boldsymbol{\Pi}$ is the identity matrix. Perturbations are partitioned in two classes: The geometric perturbations $\mathbf{p}^{(1)}=\left(p_{1}, p_{2}, p_{4}, p_{5}\right)$ and control perturbations $\mathbf{p}^{(2)}=\left(p_{3}, p_{6}\right)$. The three different workspaces $\mathcal{W}_{i}, i \in\{1,2,3\}$, to be investigated are displayed on the right graphic of Figure 4. This figure also shows the level-sets of the sensitivity index

$$
\left\|\mathbf{F}_{\mathbf{x}}(\mathbf{x}, \mathbf{q}(\mathbf{x}), \mathbf{0})^{-1} \mathbf{F}_{\mathbf{p}}(\mathbf{x}, \mathbf{q}(\mathbf{x}), \mathbf{0})\right\|,
$$

where $\mathbf{q}(\mathbf{x})$ is the inverse kinematic model. We expect from Figure 4 that $\mathcal{W}_{1}$ is the best in term of sensitivity and tolerance synthesis. 


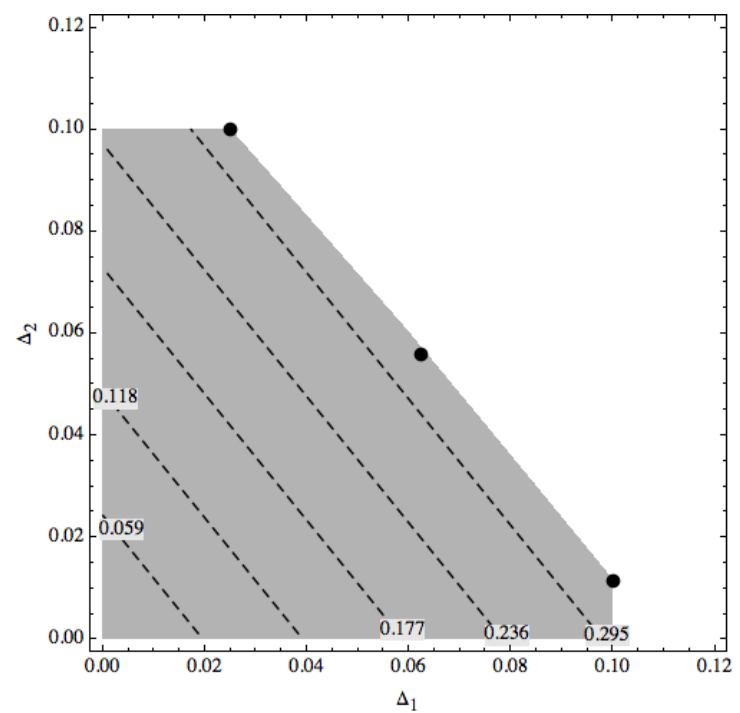

Figure 5: In gray, the perturbation domain given by Theorem 1 for $\mathcal{W}_{1}$ (note that it is truncated by $\|\Delta\| \leq \bar{\Delta}$ ). Dashed lines represent the error isocontours obtained with the linearization approximation (54).

\begin{tabular}{|c|c|c|c|c|c|}
\hline & $\kappa$ & $\chi$ & \multicolumn{2}{|c|}{$\gamma^{(1)}\left(\gamma_{0}^{(1)}\right)$} & $\gamma^{(2)}\left(\gamma_{0}^{(2)}\right)$ \\
\hline upper bound & 1.39 & 0.64 & \multicolumn{2}{|c|}{$3.5(3.01)$} & $2.97(2.43)$ \\
\hline solving time & 2.6 & 5.1 & \multicolumn{2}{|c|}{$0.5(0.1)$} & $0.2(0$ \\
\hline \multicolumn{2}{|l|}{$\Delta$} & \multicolumn{2}{|c|}{1} & certified & time \\
\hline \multicolumn{2}{|c|}{$(0.101,0.012)$} & \multicolumn{2}{|l|}{0.33} & 0.33 & 1.11 \\
\hline \multicolumn{2}{|c|}{$(0.025,0.101)$} & \multicolumn{2}{|l|}{0.32} & 0.31 & 1.23 \\
\hline \multicolumn{2}{|c|}{$(0.063,0.056)$} & \multicolumn{2}{|l|}{0.33} & 0.32 & 0.79 \\
\hline
\end{tabular}

Table 1: Results for the workspace $\mathcal{W}_{1}$ of the R $\underline{R} \underline{R} R$ robot.

The kinematic model involves only quadratic constraints, for which we can easily obtain tight Lipschitz constants for the derivatives $\lambda=2$ and $\mu=2$ without solving problems (18) and (19).

\subsubsection{Workspace $\mathcal{W}_{1}$}

The first workspace we consider is defined by $-1 \leq x_{1} \leq 0$ and $1 \leq x_{2} \leq 2$. The constants upper bounds computed for this workspace using $\bar{\Delta}=0.1$ are given in Table 1, as well as the time needed to compute them.

The corresponding error upper bound given by Theorem 1 is $\bar{\epsilon}=0.79$. The perturbation domain given by Theorem 1 is shown on Figure 5 , as well as the isocontours of the linearized error

$$
e\left(\Delta_{1}, \Delta_{2}\right) \approx 3.01 \Delta_{1}+2.43 \Delta_{2} .
$$




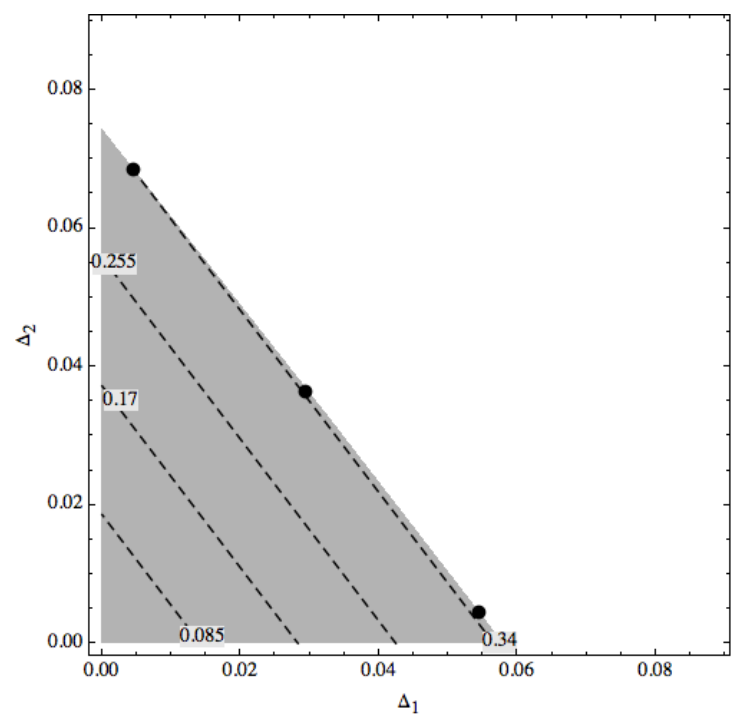

Figure 6: In gray, the perturbation domain given by Theorem 1 for $\mathcal{W}_{2}$. Dashed lines represent the error isocontours obtained with the linearization approximation (54).

The three points represent three different tradeoffs of tolerance design, which maximize the tolerances inside the perturbation domain provided by Theorem 1 . Table 1 shows for each of them the linearized error, the error upper bound obtained solving (12) using IBEX, as well as the solving time. We see that the linearized error is accurate inside the perturbation domain provided by Theorem 1 . The tolerance synthesis can therefore be performed inside this perturbation domain using the linearized errors by solving the bi-objective problem consisting of maximizing $\Delta_{1}$ and $\Delta_{2}$ subject to (56), (57) and (58), and eventually checking the chosen tolerances a posteriori solving (12).

\subsubsection{Workspace $\mathcal{W}_{2}$}

The second workspace we consider is defined by $1 \leq x_{1} \leq 2$ and $3 \leq x_{2} \leq 4$. The constants upper bounds computed for this workspace using $\bar{\Delta}=0.1$ are given in Table 2, as well as the time needed to compute them:

The corresponding error upper bound given by Theorem 1 is $\bar{\epsilon}=0.83$. The perturbation domain given by Theorem 1 is shown on Figure 6 , as well as the isocontours of the linearized error

$$
e\left(\Delta_{1}, \Delta_{2}\right) \approx 6.01 \Delta_{1}+4.57 \Delta_{2} .
$$

The three points represent three different tradeoffs of tolerance design, which maximize the tolerances inside the perturbation domain provided by Theorem 1 . Table 2 shows for each of them the linearized error, the error upper bound obtained solving (12) using IBEX, as well as the solving time. We see that the linearized error is accurate inside the perturbation domain provided by Theorem 1 . 


\begin{tabular}{|c|c|c|c|c|c|}
\hline & $\kappa$ & $\chi$ & & $\left(\gamma_{0}^{(1)}\right)$ & $\gamma^{(2)}($ \\
\hline upper bound & 2.44 & 0.61 & & $(6.01)$ & $5.48(4$ \\
\hline solving time & 0.8 & 0.1 & & $(0.1)$ & $0.1(0$ \\
\hline \multicolumn{2}{|l|}{$\overline{\Delta \Delta}$} & \multicolumn{2}{|c|}{$\overline{~ l i n e a r i z e d ~}$} & certified & time \\
\hline \multicolumn{2}{|c|}{$(0.055,0.005)$} & \multicolumn{2}{|l|}{0.35} & 0.35 & 1.13 \\
\hline \multicolumn{2}{|c|}{$(0.005,0.069)$} & \multicolumn{2}{|l|}{0.34} & 0.34 & 1.71 \\
\hline \multicolumn{2}{|c|}{$(0.03,0.037)$} & 0.35 & & 0.34 & 1.34 \\
\hline
\end{tabular}

Table 2: Results for the workspace $\mathcal{W}_{2}$ of the R $\underline{P R} \underline{P R}$ robot.

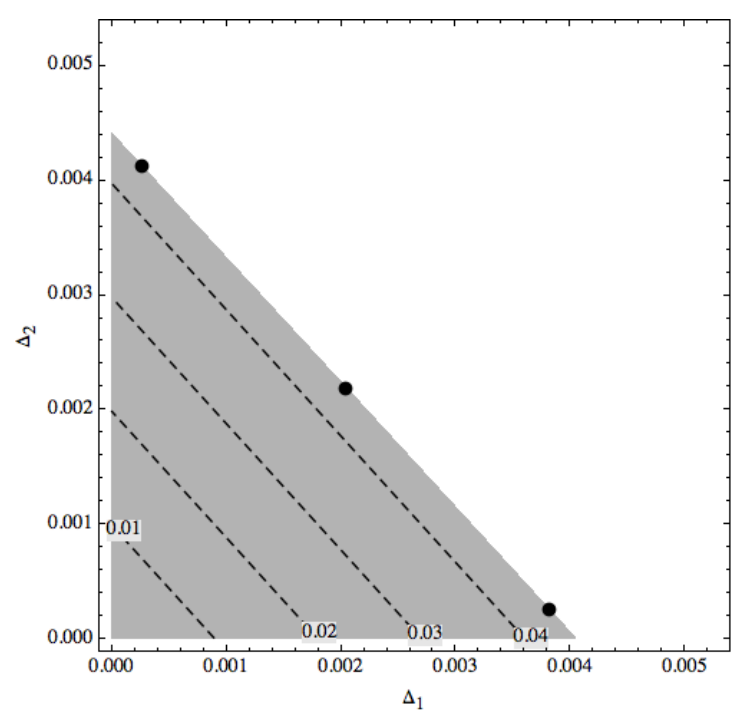

Figure 7: In gray, the perturbation domain given by Theorem 1 for $\mathcal{W}_{3}$. Dashed lines represent the error isocontours obtained with the linearization approximation 54 .

The tolerance synthesis can therefore be performed inside this perturbation domain using the linearized errors by solving the bi-objective problem consisting of maximizing $\Delta_{1}$ and $\Delta_{2}$ subject to (56), (57) and (58), and eventually checking the chosen tolerances a posteriori solving (12).

When compared with $\mathcal{W}_{1}$, we see that the linearized error is approximately twice bigger, while the perturbation domain provided by Theorem 1 is twice smaller. The workspace $\mathcal{W}_{1}$ is therefore better with respect to sensitivity and tolerance synthesis.

\subsubsection{Workspace $\mathcal{W}_{3}$}

The third workspace we consider is defined by $-0.5 \leq x_{1} \leq 0.5$ and $0.1 \leq$ $x_{2} \leq 1.1$. The constants upper bounds computed for this workspace using 


\begin{tabular}{|c|c|c|c|c|c|}
\hline & $\kappa$ & $\chi$ & \multicolumn{2}{|c|}{$\gamma^{(1)}\left(\gamma_{0}^{(1)}\right)$} & $\gamma^{(2)}\left(\gamma_{0}^{(2)}\right)$ \\
\hline upper bound & 0.05 & 5.28 & 11.6 & $(11.09)$ & $10.72(10.07)$ \\
\hline solving time & 115.8 & 0.1 & & $(0.1)$ & $0.1(0.1)$ \\
\hline \multicolumn{2}{|l|}{$\mathrm{S}_{2}$} & \multicolumn{2}{|c|}{ linearized } & certified & time \\
\hline \multicolumn{2}{|c|}{$(0.004,0.001)$} & \multicolumn{2}{|c|}{0.05} & 0.04 & 6.55 \\
\hline \multicolumn{2}{|c|}{$(0.001,0.005)$} & \multicolumn{2}{|c|}{0.05} & 0.04 & 4.37 \\
\hline & $(0.003,0.003)$ & \multicolumn{2}{|c|}{0.05} & 0.04 & 6.81 \\
\hline
\end{tabular}

Table 3: Results for the workspace $\mathcal{W}_{3}$ of the RPRPR robot.

$\bar{\Delta}=0.00 \sqrt[5]{9}$ are given in Table 3 , as well as the time needed to compute them.

The corresponding error upper bound given by Theorem 1 is $\bar{\epsilon}=0.1$. The perturbation domain given by Theorem 1 is shown on Figure 7 , as well as the linearized error $e\left(\Delta_{1}, \Delta_{2}\right) \approx 11.09 \Delta_{1}+10.07 \Delta_{2}$ isocontours.

The three points represent three different tradeoffs of tolerance design, which maximize the tolerances inside the perturbation domain provided by Theorem 1 . Table 3 shows for each of them the linearized error, the error upper bound obtained solving (12) using IBEX, as well as the solving time. We see that the linearized error is accurate inside the perturbation domain provided by Theorem 1 . The tolerance synthesis can therefore be performed inside this perturbation domain using the linearized errors by solving the bi-objective problem consisting of maximizing $\Delta_{1}$ and $\Delta_{2}$ subject to (56), (57) and (58), and eventually checking the chosen tolerances a posteriori solving (12).

When compared with $\mathcal{W}_{1}$, we see that the linearized error is now approximately 4 times bigger, while the perturbation domain provided by Theorem 1 is now 20 times smaller. The workspace $\mathcal{W}_{3}$ is therefore the worst with respect to sensitivity and tolerance synthesis.

\subsection{The $3-R \underline{P} R$ robot}

We now study the parallel robot 3-RPR with 9 perturbations, 6 of them acting on the anchor points, and 3 of them on the control. Its kinematic model $\mathbf{F}(\mathbf{x}, \mathbf{q}, \mathbf{p})$ is

$$
\begin{gathered}
\left(p_{1}-x_{1}\right)^{2}+\left(p_{2}-x_{2}\right)^{2}-\left(p_{3}+q_{1}\right)^{2} \\
\left(L+p_{4}-x_{1}-l \sin \left(\frac{\pi}{6}+x_{3}\right)\right)^{2}+\left(p_{5}-x_{2}+l \cos \left(\frac{\pi}{6}+x_{3}\right)\right)^{2} \\
-\left(p_{6}+q_{2}\right)^{2}
\end{gathered}
$$

\footnotetext{
${ }^{9}$ This workspace is closer to parallel singularities, and sensibly larger $\bar{\Delta}$ leads to perturbed poses with parallel singularities.
} 


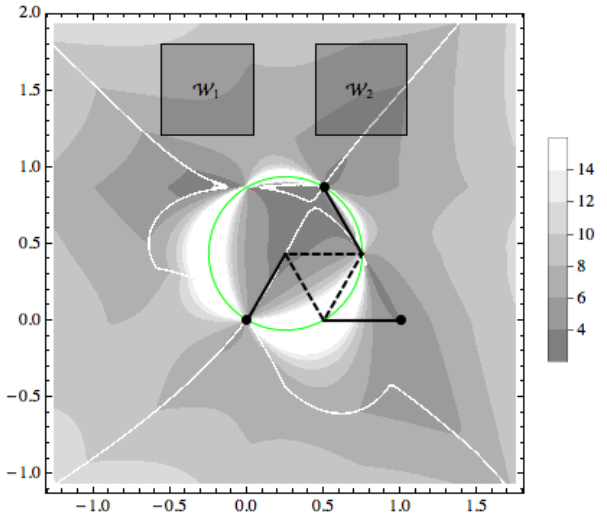

Figure 8: The 3-RR robot.

$$
\begin{aligned}
&\left(\frac{L}{2}+p_{7}-x_{1}-l \cos \left(x_{3}\right)\right)^{2}+\left(\frac{L \sqrt{3}}{2}+p_{8}-x_{2}-l \sin \left(x_{3}\right)\right)^{2} \\
&-\left(p_{9}+q_{3}\right)^{2},
\end{aligned}
$$

with $L=1$ and $l=0.5$. We study both the position error $e_{\mathrm{P}}\left(\mathbf{x}, \mathbf{x}^{\prime}\right)=$ $\left\|\boldsymbol{\Pi}_{\mathrm{P}}\left(\mathbf{x}-\mathbf{x}^{\prime}\right)\right\|=\max \left\{\left|x_{1}-x_{1}^{\prime}\right|,\left|x_{2}-x_{2}^{\prime}\right|\right\}$ and the orientation error $e_{\mathrm{O}}\left(\mathbf{x}, \mathbf{x}^{\prime}\right)=$ $\left\|\boldsymbol{\Pi}_{\mathrm{O}}\left(\mathbf{x}-\mathbf{x}^{\prime}\right)\right\|=\left|x_{3}-x_{3}^{\prime}\right|$. Perturbations are partitioned in two classes: The geometric perturbations $\mathbf{p}^{(1)}=\left(p_{1}, p_{2}, p_{4}, p_{5}, p_{7}, p_{8}\right)$ and control perturbations $\mathbf{p}^{(2)}=\left(p_{3}, p_{6}, p_{9}\right)$. The two different workspaces to be investigated are displayed on Figure 9, where only $x_{1}$ and $x_{2}$ are represented because the orientation is fixed to $x_{3}=0$ for each considered workspace. This figure also shows the level-sets of the sensitivity index

$$
\left\|\Pi \mathbf{F}_{\mathbf{x}}(\mathbf{x}, \mathbf{q}(\mathbf{x}), \mathbf{0})^{-1} \mathbf{F}_{\mathbf{p}}(\mathbf{x}, \mathbf{q}(\mathbf{x}), \mathbf{0})\right\|,
$$

where $\mathbf{q}(\mathbf{x})$ is the inverse kinematic model, $\boldsymbol{\Pi}=\boldsymbol{\Pi}_{\mathrm{P}}$ for the left graphic, and $\Pi=\Pi_{\mathrm{O}}$ for the right graphic. We expect from Figure 9 that $\mathcal{W}_{1}$ is the best in term of sensitivity and tolerance synthesis.

The kinematic model is not quadratic anymore with respect to $\mathbf{x}$, therefore we solve the following optimization problem, whose upper bound provides a second derivatives based Lipschitz constant satisfying (18):

$$
\lambda \geq \max _{\substack{\mathbf{x}, \mathbf{q}) \in \mathcal{G} \\ \mathbf{p} \in \overline{B_{\Delta}}}} \max _{i} \sum_{j k}\left|\frac{\partial f_{i}}{\partial x_{j} x_{k}}(\mathbf{x}, \mathbf{q}, \mathbf{p})\right| .
$$

The kinematic model is quadratic with respect to $\mathbf{p}$, and we can obtain the sharp Lipschitz constant $\mu=2$.

IBEX resolution timings are quite higher than for the RPRPR robot, because more variables are involved and expressions are more complex. On the other hand, the standard version of IBEX has been used, and specific tuning may turn out to significantly reduce the resolution timings. 

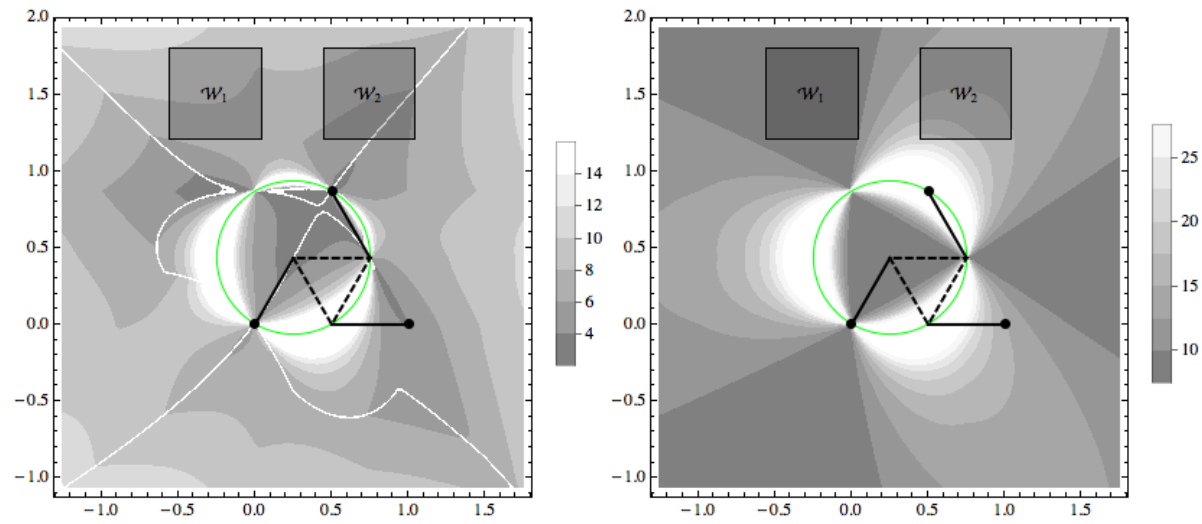

Figure 9: Sensitivity indices and the two workspaces considered for the 3-RPR robot (the green circle represents parallel singularities; the white curves are drawing artefacts due to the non-differentiability of the sensitivity index). The left graphic and the right graphics correspond to position and orientation sensitivities respectively.

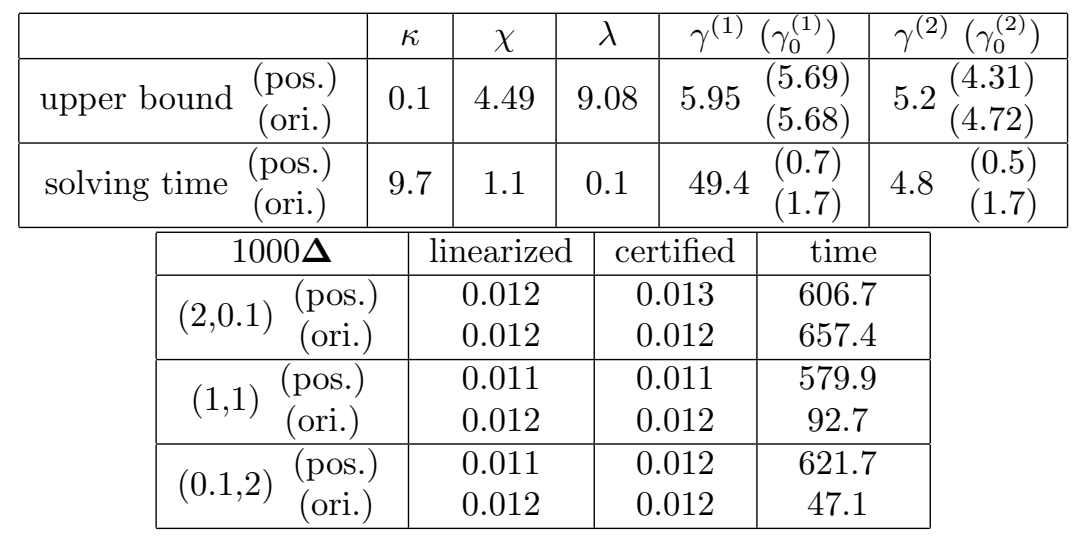

Table 4: Results for the workspace $\mathcal{W}_{1}$ of the $3-\mathrm{RPR}$ robot.

\subsubsection{Workspace $\mathcal{W}_{1}$}

The first workspace we consider is defined by $-0.55 \leq x_{1} \leq 0.05,1.2 \leq x_{2} \leq$ 1.8 and $x_{3}=0$. The constants upper bounds computed for this workspace using $\bar{\Delta}=0.01$ are given in Table 4 , as well as the time needed to compute them.

The corresponding error upper bound given by Theorem 1 is $\bar{\epsilon}=0.03$. The perturbation domain given by Theorem 1 is shown on Figure 10, as well as the isocontours of the linearized position (blue dashed lines) and orientation (green dashed lines) errors

$$
\begin{aligned}
& e_{\mathrm{P}}\left(\Delta_{1}, \Delta_{2}\right) \approx 5.69 \Delta_{1}+4.31 \Delta_{2} \\
& e_{\mathrm{O}}\left(\Delta_{1}, \Delta_{2}\right) \approx 5.68 \Delta_{1}+4.72 \Delta_{2} .
\end{aligned}
$$




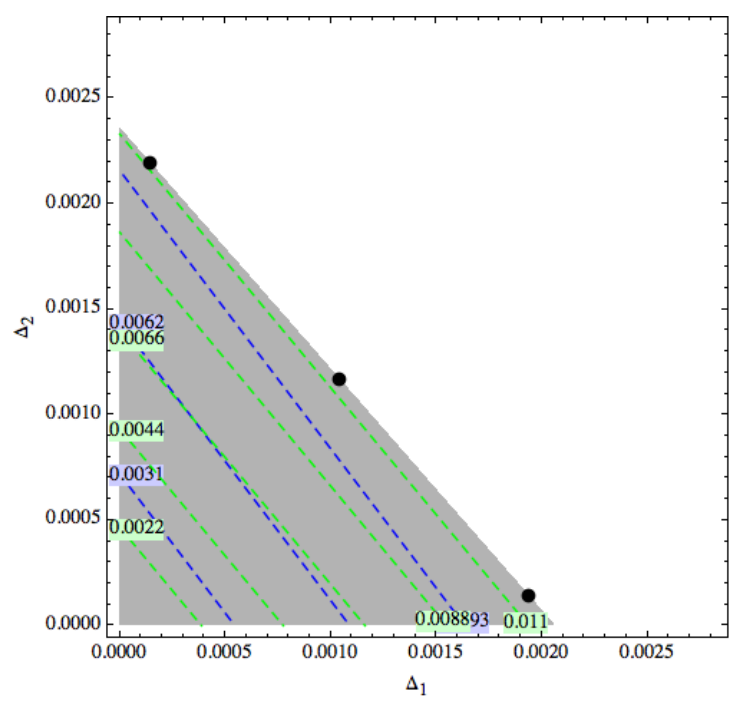

Figure 10: In gray, the perturbation domain given by Theorem 1 for $\mathcal{W}_{1}$. Dashed lines represent the error isocontours obtained with the linearization approximation 54 .

The three points represent three different tradeoffs of tolerance design, which maximize the tolerances inside the perturbation domain provided by Theorem 1 . Table 4 shows for each of them the linearized error, the error upper bound obtained solving $(12$ using IBEX, as well as the solving time. We see that the linearized error is accurate inside the perturbation domain provided by Theorem 1 . The tolerance synthesis can therefore be performed inside this perturbation domain using the linearized errors by solving the bi-objective problem consisting of maximizing $\Delta_{1}$ and $\Delta_{2}$ subject to (56), (57) and (58), and eventually checking the chosen tolerances a posteriori solving (12).

\subsubsection{Workspace $\mathcal{W}_{2}$}

The second workspace we consider is defined by $0.45 \leq x_{1} \leq 1.05,1.2 \leq$ $x_{2} \leq 1.8$ and $x_{3}=0$. The constants upper bounds computed for this workspace using $\bar{\Delta}=0.01$ are given in Table 5 , as well as the time needed to compute them.

The corresponding error upper bound given by Theorem 1 is $\bar{\epsilon}=0.03$. The perturbation domain given by Theorem 1 is shown on Figure 11, as well as the isocontours of the linearized position (blue dashed lines) and orientation (green dashed lines) errors

$$
\begin{aligned}
e_{\mathrm{P}}\left(\Delta_{1}, \Delta_{2}\right) & \approx 4.7 \Delta_{1}+3.85 \Delta_{2} \\
e_{\mathrm{O}}\left(\Delta_{1}, \Delta_{2}\right) & \approx 12.16 \Delta_{1}+9.13 \Delta_{2} .
\end{aligned}
$$

The three points represent three different tradeoffs of tolerance design, which maximize the tolerances inside the perturbation domain provided by Theorem 1 . 


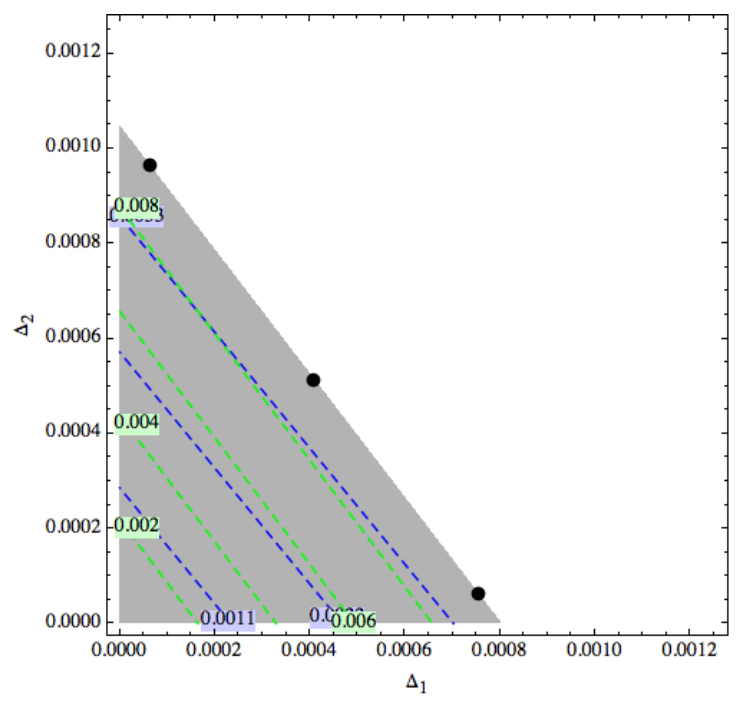

Figure 11: In gray, the perturbation domain given by Theorem 1 for $\mathcal{W}_{2}$. Dashed lines represent the error isocontours obtained with the linearization approximation (54).

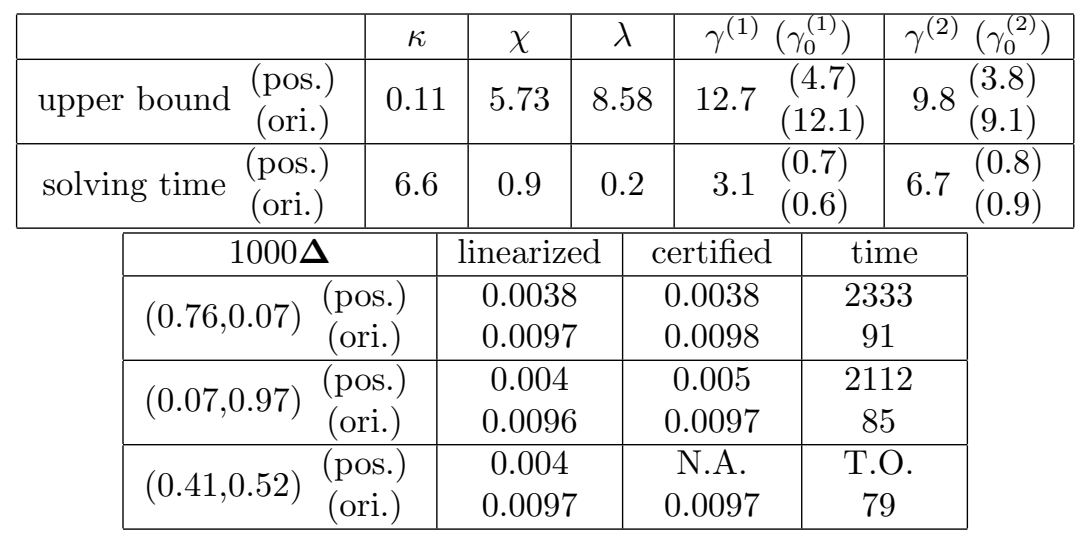

Table 5: Results for the workspace $\mathcal{W}_{2}$ of the 3-RPR robot.

Table 5 shows for each of them the linearized error, the error upper bound obtained solving (12) using IBEX, as well as the solving time. We see that the linearized error is accurate inside the perturbation domain provided by Theorem 1 . The tolerance synthesis can therefore be performed inside this perturbation domain using the linearized errors by solving the bi-objective problem consisting of maximizing $\Delta_{1}$ and $\Delta_{2}$ subject to (56), (57) and (58), and eventually checking the chosen tolerances a posteriori solving (12).

As predicted by Figure 9, we see that the position linearized error is approx- 
imately the same as $\mathcal{W}_{1}$ (actually slightly better for $\mathcal{W}_{2}$ ) but the orientation error is now twice worst. The perturbation domain provided by Theorem 1 is twice smaller as well. The workspace $\mathcal{W}_{1}$ is therefore better with respect to sensitivity and tolerance synthesis.

\section{Conclusion}

The three step approach proposed in this paper was successfully applied to a 2-DOF and a 3-DOF parallel robot. Theorem 1 was able to compute safe domains of perturbations at Step 1, whose size is a few percents of the length of the longest link in the case of the 2-DOF robot, and a few per thousand of the length of the longest link in the case of the 3-DOF robot, which are realistic for the purpose of synthesizing tolerances. The approximate linearization computed at Step 2 provided accurate approximations, which have been confirmed by the sharp upper bound computed at Step 3.

However, these preliminary experiments also clearly show that the more complex the robot, the smaller the safe domain of perturbation computed by Theorem 1 and the longer computation time. Therefore, those two issues should be solved for the method to be applicable to robots with more DOF. First, larger safe domains of perturbations could be obtained by improving the expressions of problems (14), (15), and (16), where there is a loss of correlation due to the fact that global maximum may be reached at different poses in the workspace, as well as by investigating the usage of other norms that can enlarge the convergence domain provided by Kantorovich theorem. Second, improving computation timings for more complex robots entails tuning and specializing the optimization software IBEX, as well as handling numerically the inverse Jacobian, which is currently formally inverted.

\section{References}

[1] J. Wang, O. Masory, On the accuracy of a stewart platform - part i, the effect of manufacturing tolerances., in: Proceedings of the IEEE International Conference on Robotics Automation, ICRA'93, 1993.

[2] H. Kim, Y. Choi, The kinematic error bound analysis of the stewart platform, Journal of Robotic Systems 17 (2000) 63-73.

[3] H. Kim, L.-W. Tsai, Design optimization of a cartesian parallel manipulator, ASME Journal of Mechanical Design 125 (2003) 43-51.

[4] C. Han, J. Kim, J. Kim, P. F.C., Kinematic sensitivity analysis of the 3-upu parallel mechanism, Mechanism and Machine Theory 37 (2002) 787-798.

[5] K.-C. Fan, H. Wang, J.-W. Zhao, T.-H. Chang, Sensitivity analysis of the 3 -prs parallel kinematic spindle platform of a serial-parallel machine tool, International Journal of Machine Tools and Manufacture 43 (2003) 15611569. 
[6] M. Verner, X. Fengfeng, C. Mechefske, Optimal calibration of parallel kinematic machines, ASME Journal of Mechanical Design 127 (2005) 62-69.

[7] S. Caro, F. Bennis, P. Wenger, Tolerance synthesis of mechanisms : A robust design approach, ASME Journal of Mechanical Design 127 (2005) 86-94, hal-00463707.

[8] J. Ryu, J. Cha, Volumetric error analysis and architecture optimization for accuracy of hexaslide type parallel manipulators, Mechanism and Machine Theory 38 (2003) 227-240.

[9] H. Liu, T. Huang, D. Chetwynd, A general approach for geometric error modeling of lower mobility parallel manipulators, ASME Journal of Mechanisms and Robotics 3 (2) (2011) 0210131-02101313.

[10] S. Briot, I. A. Bonev, Are parallel robots more accurate than serial robots, Transactions of the Canadian Society for Mechanical Engineering 31 (4) (2007) 445-455.

[11] L. Rolland, Outils algbriques pour la rsolution de problmes gomtriques et l'analyse de trajectoire de robots parallles prvus pour des applications haute cadence et grande prcision, Ph.D. thesis, Universit Nancy 1 (2002).

[12] A. Patel, K. Ehmann, Volumetric error analysis of a stewart platform based machine tool, In Annals of the CIRP 46 (1997) 287-290.

[13] A. Yu, I. Bonev, P. Zsombor-Murray, Geometric approach to the accuracy analysis of a class of 3-dof planar parallel robots, Mechanism and Machine Theory 43 (3) (2009) 364-375.

[14] S. Bai, S. Caro, Design and analysis of a 3-ppr planar robot with u-shape base, in: Proceedings of the 14th International Conference on Advanced Robotics, Munich, Germany, 2009.

[15] J. Merlet, Parallel robots, Kluwer, Dordrecht, 2000.

[16] S. Caro, P. Wenger, F. Bennis, D. Chablat, Sensitivity analysis of the orthoglide, a 3-dof translational parallel kinematic machine, ASME Journal of Mechanical Design 128 (2006) 392-402.

[17] S. Caro, N. Binaud, P. Wenger, Sensitivity Analysis of 3-RPR Planar Parallel Manipulators, ASME Journal of Mechanical Design 131 (2009) 121005$1-121005-13$.

[18] M. Tannous, S. Caro, A. Goldsztejn, Sensitivity analysis of parallel manipulators using an interval linearization method, Mechanism and Machine Theory 71 (0) (2014) 93 - 114.

[19] N. Berger, R. Soto, A. Goldsztejn, S. Caro, P. Cardou, Finding the Maximal Pose Error in Robotic Mechanical Systems Using Constraint Programming, in: Proceedings of IEA-AIE 2010, Vol. 6096 of LNAI, 2010, pp. 82-91. 
[20] W. Khalil, E. Dombre, Modeling, Identification and Control of Robots, 3rd Edition, Taylor \& Francis, Inc., Bristol, PA, USA, 2002.

[21] H. Bamberger, A. Wolf, M. Shoham, Assembly mode changing in parallel mechanisms, Robotics, IEEE Transactions on 24 (4) (2008) 765-772.

[22] S. Briot, V. Arakelian, Optimal force generation in parallel manipulators for passing through the singular positions, Int. J. Rob. Res. 27 (8) (2008) 967-983.

[23] J. M. Ortega, The Newton-Kantorovich Theorem, The American Mathematical Monthly 75 (6) (1968) 658-660.

[24] W. Gragg, R. Tapia, Optimal Error Bounds for the Newton-Kantorovich Theorem, SIAM Journal on Numerical Analysis 11 (1) (1974) 10-13.

[25] P. G. Ciarlet, C. Mardare, On the Newton-Kantorovich Theorem, Analysis and Applications 10 (03) (2012) 249-269.

[26] A. Goldsztejn, L. Granvilliers, A New Framework for Sharp and Efficient Resolution of NCSP with Manifolds of Solutions, Constraints 15 (2) (2010) 190-212.

[27] B. Martin, A. Goldsztejn, C. Jermann, L. Granvilliers, Certified Parallelotope Continuation for One-Manifolds, SIAM Journal On Numerical Analysis 51 (6) (2013) 3373-3401.

[28] D. Ishii, A. Goldsztejn, C. Jermann, Interval-Based Projection Method for Under-Constrained Numerical Systems, Constraints 17 (4) (2012) 432-460.

[29] S. Caro, D. Chablat, A. Goldsztejn, D. Ishii, C. Jermann, A Branch and Prune Algorithm for the Computation of Generalized Aspects of Parallel Robots, Artificial Intelligence 221 (2014) 34-50.

[30] A. Neumaier, S. Zuhe, The Krawczyk operator and Kantorovich's theorem, Journal of Mathematical Analysis and Applications 149 (2) (1990) 437 443.

[31] G. Chabert, L. Jaulin, Contractor Programming, Artif. Intell. 173 (11) (2009) 1079-1100.

[32] G. Trombettoni, I. Araya, B. Neveu, G. Chabert, Inner regions and interval linearizations for global optimization., in: W. Burgard, D. Roth (Eds.), AAAI, AAAI Press, 2011.

[33] I. Araya, G. Trombettoni, B. Neveu, G. Chabert, Upper Bounding in Inner Regions for Global Optimization Under InequalityConstraints, J. Global Optimization 60 (2) (2014) 145-164.

[34] K. Miettinen, Nonlinear Multiobjective Optimization, Kluwer, 1998.

[35] Ehrgott, Multicriteria Optimization, Springer, 2005. 


\section{Appendix A. Kantorovich theorem}

Theorem 2 ([24]). Let $D_{0}$ be an open convex subset of $\mathbb{R}^{n}$, and $\mathbf{h}: D_{0} \rightarrow \mathbb{R}^{n}$ be differentiable on $D_{0}$ with its derivative $\mathbf{H}_{\mathbf{x}}$ satisfying

$$
\left\|\mathbf{H}_{\mathbf{x}}(\mathbf{x})-\mathbf{H}_{\mathbf{x}}\left(\mathbf{x}^{\prime}\right)\right\| \leq \lambda\left\|\mathbf{x}-\mathbf{x}^{\prime}\right\|
$$

for all $\mathbf{x}, \mathbf{x}^{\prime} \in D_{0}^{10}$. Assume that $\mathbf{x}_{0} \in D_{0}$ is such that $\mathbf{\Gamma}_{0}:=\mathbf{H}_{\mathbf{x}}\left(\mathbf{x}_{0}\right)^{-1}$ exists,

$$
\left\|\boldsymbol{\Gamma}_{0}\right\| \leq \chi,\left\|\boldsymbol{\Gamma}_{0} \mathbf{h}\left(\mathbf{x}_{0}\right)\right\| \leq \delta, h:=2 \chi \lambda \delta \leq 1
$$

and

$$
B\left(\mathbf{x}_{0}, t^{*}\right) \subseteq D_{0}, t^{*}:=\frac{2}{h}(1-\sqrt{1-h}) \delta
$$

Then:

1. The Newton iterates $\mathbf{x}_{n+1}=\mathbf{x}_{n}-\mathbf{H}_{\mathbf{x}}\left(\mathbf{x}_{n}\right)^{-1} \mathbf{h}\left(\mathbf{x}_{n}\right)$ exists and $\mathbf{x}_{n} \in B\left(\mathbf{x}_{0}, t^{*}\right) \subseteq$ $D_{0}$ for all $n \geq 0$.

2. $\mathbf{x}^{*}:=\lim \mathbf{x}_{n}$ exists, $\mathbf{x}^{*} \in \bar{B}\left(\mathbf{x}_{0}, t^{*}\right) \subseteq \bar{D}_{0}$ and $\mathbf{h}\left(\mathbf{x}^{*}\right)=\mathbf{0}$.

3. $\mathbf{x}^{*}$ is the only solution of $\mathbf{h}(\mathbf{x})=\mathbf{0}$ in the set

$$
B\left(\mathbf{x}_{0}, t^{\prime}\right) \cap D_{0}, t^{\prime}:=\frac{2}{h}(1+\sqrt{1-h}) \delta
$$

if $h<1$, and in $\bar{B}\left(\mathbf{x}_{0}, t^{\prime}\right)$ if $h=1$.

Kantorovich theorem involves three constants: $\chi$ and $\delta$ are directly related to the computation of the first Newton step applied to solve the system ( $\delta$ is an upper bound of the norm of the first Newton step, $\chi$ provides a distance to singularity of the Jacobian matrix), while $\lambda$ is related to the strength of nonlinearity of the system (the system is linear for $\lambda=0$, while the derivative may change quicker as $\lambda$ increases). Kantorovich theorem provides both an existence domain $\bar{B}\left(\mathbf{x}_{0}, t^{*}\right)$ and a uniqueness domain $B\left(\mathbf{x}_{0}, t^{\prime}\right) \cap D_{0}$. Of course, a smaller existence domain is more accurate, while a larger uniqueness domain is better. The following lemma clarifies the behavior of $t^{*}$ and $t^{\prime}$ with respect to Kantorovich constants.

Lemma 1. $t^{*}$ is increasing w.r.t. each Kantorovich constants, while $t^{\prime}$ is decreasing w.r.t. each Kantorovich constants. Furthermore, $t^{*}$ is strictly increasing w.r.t. $\delta$, while $t^{\prime}$ is strictly decreasing w.r.t. $\delta$.

Proof. The proof is simply conducted by studying the sign of the derivatives

\footnotetext{
${ }^{10}$ It is then elementary, using completeness, that $\mathbf{h}$ and $\mathbf{H}_{\mathbf{x}}$ have unique continuous extensions to the closure $\bar{D}_{0}$ of $D_{0}$, and $\mathrm{A} .10$ is satisfied for all $\mathbf{x}, \mathbf{x}^{\prime} \in \bar{D}_{0}$.
} 
of $t^{*}$ and $t^{\prime}$ with respect to Kantorovich constants. E.g.,

$$
\begin{aligned}
\frac{\partial t^{*}}{\partial \chi} & =\frac{\delta}{\chi \sqrt{1-h}}-\frac{1-\sqrt{1-h}}{\lambda \chi^{2}} \\
& =\frac{1-\frac{1}{2} h-\sqrt{1-h}}{\lambda \chi \sqrt{1-h}} \\
& \geq \frac{1-h-\sqrt{1-h}}{\lambda \chi \sqrt{1-h}} \\
& \geq 0 .
\end{aligned}
$$

The other cases are conducted similarly.

As a consequence, the worst existence and uniqueness domains are obtained for $h=2 \chi \lambda \delta=1$, in which case $t^{*}=t^{\prime}=2 \delta=\frac{1}{\chi \lambda}$. This is formalized in Equation A.11) and Equation A.13). The following corollary simplifies Theorem 2 by fixing $D_{0}$ so that $B\left(\mathbf{x}_{0}, t^{*}\right) \subseteq D_{0}$ and provides a less accurate but easier to use uniqueness domain by using the smaller uniqueness domain for all cases $h=1$ and $h<1$. Furthermore, aiming an application to a parametric system of equations, two upper bounds $r$ and $\delta$ for the first Newton step are used: The first being meant to be crude (independent of the parameters), the second accurate (depending on the parameters).

Corollary 1. Let $\mathbf{h}: D \subseteq \mathbb{R}^{n} \rightarrow \mathbb{R}^{n}$ and $\mathbf{x}_{0} \in D$ be such that $\boldsymbol{\Gamma}_{0}:=\mathbf{H}_{\mathbf{x}}\left(\mathbf{x}_{0}\right)^{-1}$ exists. Suppose that

$$
\left\|\boldsymbol{\Gamma}_{0}\right\| \leq \chi,\left\|\boldsymbol{\Gamma}_{0} \mathbf{h}\left(\mathbf{x}_{0}\right)\right\| \leq \delta .
$$

For a given $r \geq \delta$, consider $D_{0}:=B\left(x_{0},(2 r)^{+}\right)$, where $(2 r)^{+}$is any number strictly greater than $2 r$, and suppose that $\mathbf{h}$ is defined and differentiable in $D_{0}$ with

$$
\left\|\mathbf{H}_{\mathbf{x}}(\mathbf{x})-\mathbf{H}_{\mathbf{x}}\left(\mathbf{x}^{\prime}\right)\right\| \leq \lambda\left\|\mathbf{x}-\mathbf{x}^{\prime}\right\|
$$

for all $\mathbf{x}, \mathbf{x}^{\prime} \in D_{0}$. Finally assume that $h:=2 \chi \lambda \delta \leq 1$. Then $\mathbf{h}(\mathbf{x})=\mathbf{0}$ has one solution $\mathbf{x}^{*}$ inside $\bar{B}\left(\mathbf{x}_{0}, t^{*}\right), t^{*}:=\frac{2}{h}(1-\sqrt{1-h}) \delta$, which is unique inside $\bar{B}\left(\mathbf{x}_{0}, \min \left\{2 r, \frac{1}{\chi \lambda}\right\}\right) \supseteq \bar{B}\left(\mathbf{x}_{0}, t^{*}\right)$.

Proof. We first need to prove that $B\left(\mathbf{x}_{0}, t^{*}\right) \subseteq D_{0}$, i.e., $t^{*} \leq(2 r)^{+}$, so that all hypotheses of Theorem 2 are satisfied. By Lemma $1, t^{*}$ is increasing w.r.t. $\chi$ and $\lambda$, therefore for an arbitrary fixed $\delta$ we have

$$
\max _{\substack{\chi, \lambda \geq 0 \\ 2 \chi \lambda \delta \leq 1}} t^{*}(\chi, \lambda, \delta)=\max _{\substack{\chi, \lambda \geq 0 \\ 2 \chi \lambda \delta=1}} t^{*}(\chi, \lambda, \delta)=\max _{\substack{\chi, \lambda \geq 0 \\ 2 \chi \lambda \delta=1}} 2 \delta=2 \delta \leq 2 r .
$$

Therefore, Theorem 2 applies and proves the existence of a solution $\mathbf{x}^{*}$ inside $\bar{B}\left(\mathbf{x}_{0}, t^{*}\right) \subseteq \bar{D}_{0}$.

The uniqueness of the solution inside $\bar{B}\left(\mathbf{x}_{0}, \min \left\{2 r, \frac{1}{\chi \lambda}\right\}\right)$ is proved in two steps: First, if $h=1$ then $t^{\prime}=\frac{1}{\chi \lambda}=2 \delta$, which is less than $2 r$ by hypothesis. 
Therefore $\min \left\{2 r, \frac{1}{\chi^{\lambda}}\right\}=\frac{1}{\chi^{\lambda}}$ and Theorem 2 proves the uniqueness inside

$$
\bar{B}\left(\mathbf{x}_{0}, t^{\prime}\right)=\bar{B}\left(\mathbf{x}_{0}, \frac{1}{\chi \lambda}\right)=\bar{B}\left(\mathbf{x}_{0}, \min \left\{2 r, \frac{1}{\chi \lambda}\right\}\right) .
$$

Second, if $0 \leq h<1$ then Theorem 2 proves uniqueness inside $B\left(\mathbf{x}_{0}, t^{\prime}\right) \cap D_{0}$. We now prove that $0 \leq h<1$ entails $t^{\prime}>\frac{1}{\chi \lambda}$ : By hypothesis $h<1$, or equivalently $\delta<\frac{1}{2 \chi \lambda}$. By Lemma 1, $t^{\prime}$ is strictly decreasing w.r.t. $\delta$, therefore

$$
t^{\prime}(\chi, \lambda, \delta)>t^{\prime}\left(\chi, \lambda, \frac{1}{2 \chi \lambda}\right)=\frac{1}{\chi \lambda} .
$$

As a consequence,

$$
B\left(\mathbf{x}_{0}, t^{\prime}\right) \cap D_{0} \supseteq \bar{B}\left(\mathbf{x}_{0}, \frac{1}{\chi \lambda}\right) \cap \bar{B}\left(\mathbf{x}_{0}, 2 r\right)=\bar{B}\left(\mathbf{x}_{0}, \min \left\{2 r, \frac{1}{\chi \lambda}\right\}\right),
$$

the first inclusion holding because $t^{\prime}>\frac{1}{\chi^{\lambda}}$ and $D_{0}=B\left(\mathbf{x}_{0},(2 r)^{+}\right) \supseteq \bar{B}\left(\mathbf{x}_{0}, 2 r\right)$.

Of course, Corollary 1 can also be applied to a non-parametric system of equations by setting $r=\bar{\delta}$, in which case the uniqueness domain $\bar{B}\left(\mathbf{x}_{0}, \min \left\{2 r, \frac{1}{\chi^{\lambda}}\right\}\right)$ can be simplified to $\bar{B}\left(\mathbf{x}_{0}, 2 \delta\right)$ (since $\left.2 r=2 \delta \leq \frac{1}{\chi^{\lambda}}\right)$.

\section{Appendix B. Minibex code for the PRRP robot}

Minibex is a simple language that allows describing constrained optimization problems to be solved by IBEX. Such files can be solved directly by IBEX. In the Minibex codes below, the maximization of each quantity is performed by minimizing its opposite.

Appendix B.1. Minibex code for $\kappa$

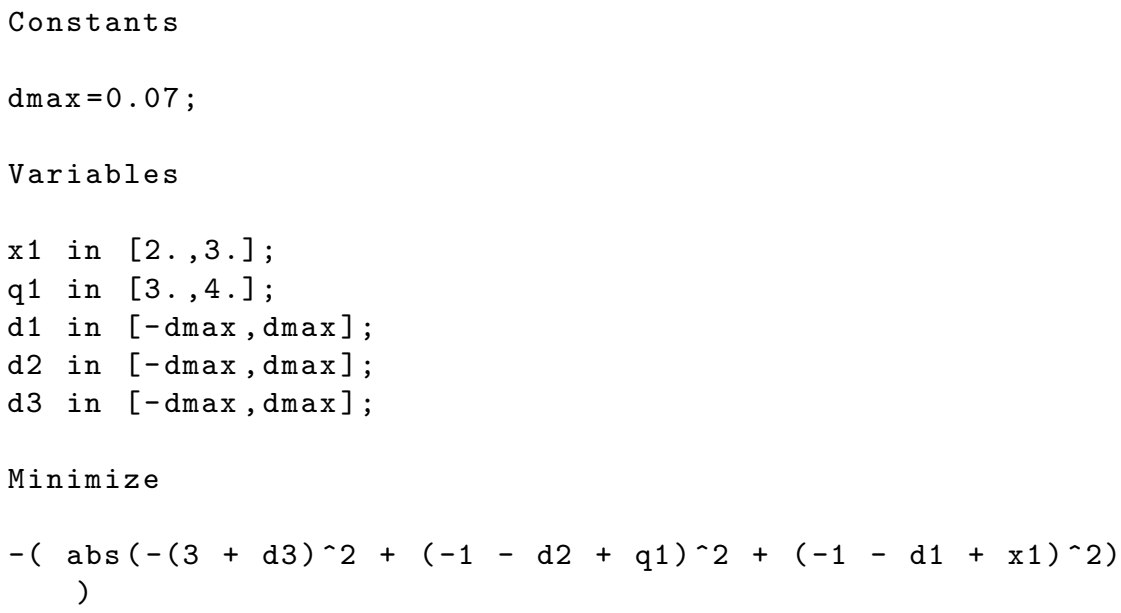




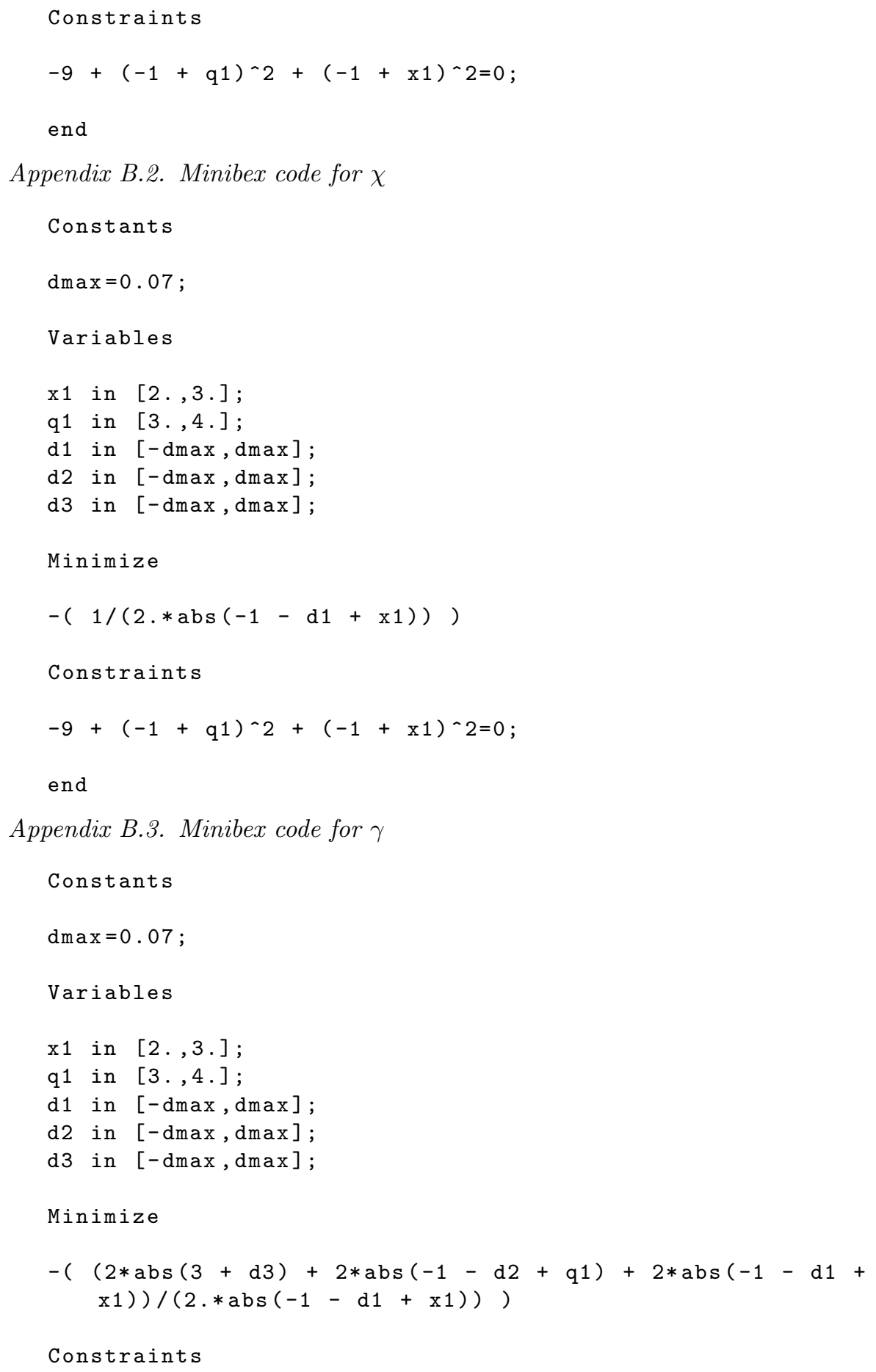




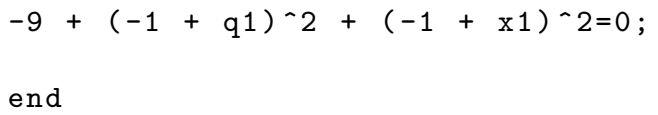

Appendix B.4. Minibex code for the sharp error estimate

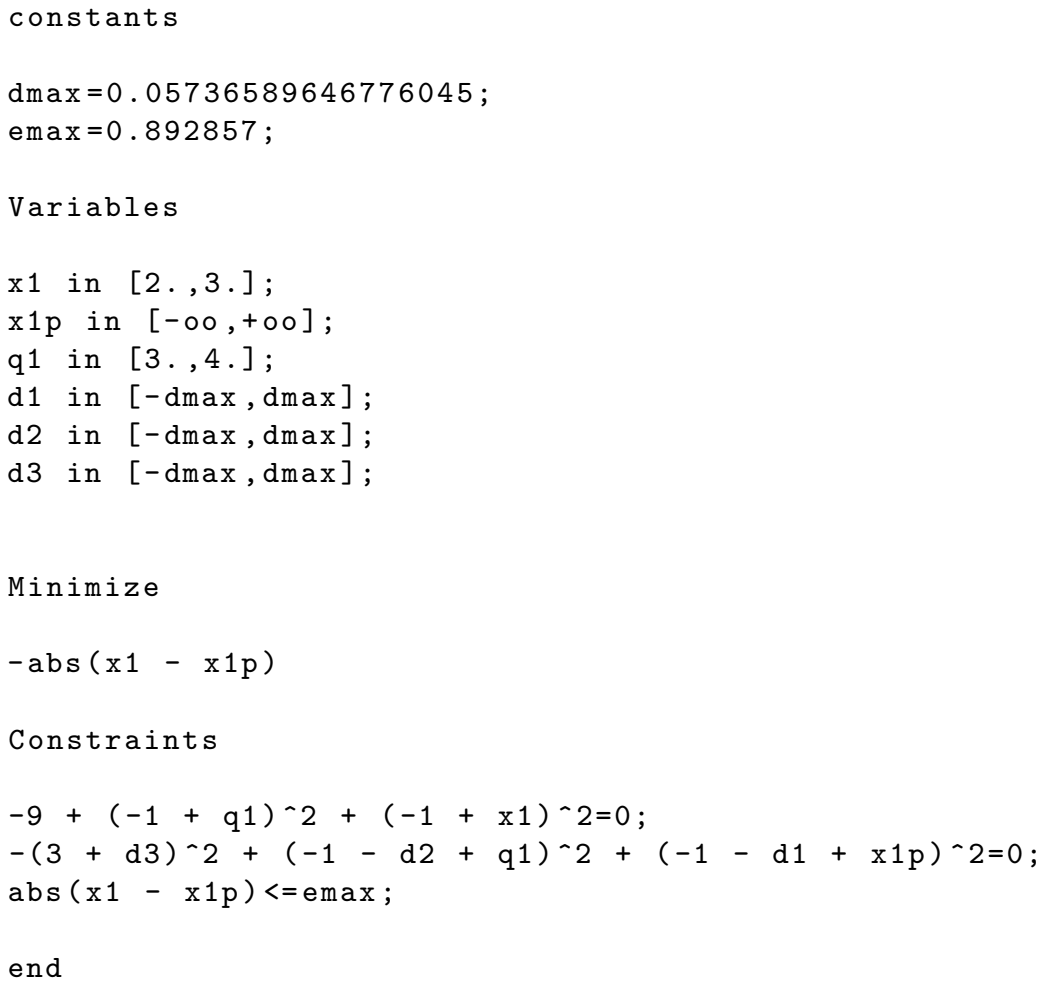

